\title{
A topological version of a theorem of Veech and almost simple flows
}

\author{
ELI GLASNER \\ School of Mathematical Sciences, The Raymond and Beverly Sackler Faculty of Exact \\ Sciences, Tel Aviv University, Tel Aviv, Israel
}

(Received 22 July 1988)

\begin{abstract}
Almost simple (AS) minimal flows are defined and it is shown that any factor map of an AS flow is, up to almost 1-1 equivalence, a group factor. An analogous theorem for metric, regular, point distal extensions is proved. In particular a theorem of Gottschalk is strengthened to show that any regular, point distal, metric flow is equicontinuous. When the acting group $T$ is commutative it is shown that every proper minimal joining of an AS flow $X$ and a minimal flow $Y$, is, up to almost 1-1 extensions, the relative product of $X$ and $Y$ over a common factor which is a group factor of $X$.
\end{abstract}

\section{Introduction}

An ergodic process $(X, T, m)$, where $X$ is compact metric and $T$ a Borel one to one measure preserving map of $X$ into itself, is called (two-fold) simple, if every ergodic self joining of $(X, T, m)$ is either $m \times m$ or a measure of the form $(\operatorname{Id} \times S) \Delta=$ $\Delta_{S}$ where $S$ is a Borel automorphism of $(X, T, m)$ and $\Delta$ is the probability measure on $X \times X$ defined by: $\int f d \Delta=\int f(x, x) d m(x)$. The main result of [V2] is that for a simple process every non-trivial factor $(X, T, m) \stackrel{m}{\rightarrow}\left(X^{\prime}, T^{\prime}, m^{\prime}\right)$ is a compact group factor; i.e., there exists a compact group $K$ of automorphisms of $(X, T, m)$ such that $X^{\prime}=X / K$ and $m^{\prime}, T^{\prime}$ are the induced measure and measure preserving transformation, respectively. The crucial device in proving this theorem is the following easy version of a theorem of $\mathrm{A}$. Weil [V2]

THEOREM. If $K$ is a Polish topological group admitting a left invariant Borel probability measure, then $K$ is compact.

Let $(X, T, m)$ be (two fold) simple, $[\mathbf{J}-\mathbf{R}]$, then $A$, its group of automorphisms is a Polish topological group under the weak topology. If $(X, T, m) \stackrel{m}{\rightarrow}\left(X^{\prime}, T^{\prime}, m^{\prime}\right)$ is a factor map then simplicity of $(X, T, m)$ implies that the subgroup $K=$ $\{S \in A: \pi \circ S=\pi\}$ admits an invariant probability measure and by Weil's theorem is therefore compact. It then follows that $\pi$ is $K$-extension.

When we look for a theorem analogous to that of Veech in topological dynamics, we are faced with two problems. The first is to find an appropriate notion of topological simplicity and the second is to find a substitute for Weil's theorem. Our starting point for the latter problem will be the following theorem of Gottschalk, [Go]. 
TheOREM. Let $(X, T)$ be a topologically ergodic metric flow, where $T$ is an abelian group. If the group Aut $(X, T)$ acts transitively on $X$ then $(X, T)$ is equicontinuous.

As was observed by J. Auslander [A], Gottschalk's theorem, for minimal flows, can be reformulated as the statement that a metric distal regular $T$-flow for abelian $T$ is equicontinuous. In $\S 3$ we shall show that the assumption of commutativity in Gottschalk's theorem is not necessary; any metric regular, flow is equicontinuous (Proposition 3.1). (This was proved independently and earlier by J. Auslander [A1].) In fact we shall show that moreover any regular, open, point distal homomorphism of a metric minimal flow is necessarily a group extension (Theorem 4.7 ). We consider these theorems as topological counterparts of Weil's theorem.

Our definition for topological simplicity, which we call Almost Simple (AS), is motiviated by A. del-Junco's definition of topological almost minimal self-joinings (AMSJ), $[\mathbf{J}]$. We show that AS is an invariant of almost 1-1 extensions and that a weak-mixing group extension of AMSJ is AS. Well known results imply now that any non-trivial factor map $X \stackrel{\pi}{\rightarrow} Y$ of an AS flow $X$ is necessarily point distal (Proposition 1.7). In $\S 4$ we get the stronger result that up to almost 1-1 extensions $\pi$ is actually a group extension (Theorem 4.5 ). The main proposition for the proofs of both Theorems 4.5 and 4.7 is proved in $\S 2$ (Proposition 2.5). Actually, among the assumptions in Proposition 2.5, we include one (that $\tilde{\pi}$ is $\mathrm{Bc}$ ) which we do not have when we come to apply it in the proofs of Theorems 4.5 and 4.7. However, in some special cases, namely when $\pi$ is distal and when $X$ is not weakly mixing, this assumption does hold and in $\S 3$ we do use Proposition 2.5 in proving the corresponding results in these special cases (Propositions 3.1 and 3.2). In this way, we hope, the main ideas and also the exposition are made clear and easier to follow. Finally in $\$ 4$ we discuss the technical changes which we need to introduce into Proposition 2.5 so that Theorems 4.5 and 4.7 can be deduced. This way of proof necessitates a certain amount of repetition.

In [J-R] the following theorem is proved for ergodic measure preserving actions $(X, \mu, T)$ and $(Y, \nu, T)$.

THEOREM. If $(X, \mu)$ is simple then every ergodic joining of $(X, \mu)$ and $(Y, \nu)$ which is not product measure is the projection on $X \times Y$ of the relatively independent joining of $\left(X^{n}, \mu^{n}\right)$ and $(Y, \nu)$ over $(X / K)^{n \odot}$, for some compact subgroup $K$ of $C(X, \mu T)$ and some factor map $\varphi: Y \rightarrow(X / K)^{n \odot}$.

Here $C(X, \mu, T)$ is the group of automorphisms of $(X, \mu, T), X / K$ is the factor of $(X, \mu, T)$ corresponding to $K$ and $(X / K)^{n \odot}$ is $(X / K)^{n} / S_{n}$ where $S_{n}$ is the group of permutations on the coordinates of $(X / K)^{n}$.

In $\$ 6$ we obtain, using Theorem 4.5 a topological analogue for this theorem. Simple is replaced by AS and ergodic joining is replaced by minimal joining, i.e., a minimal subset of $X \times Y$. We are not able to prove our theorem for general $T$ and have to restrict it to abelian $T$. Under these assumptions we prove (Theorem 6.1 ) that any proper minimal joining $L \subset X \times Y$ is, up to almost 1-1 extensions, the relative product of $X$ and $Y$ over a common factor which is, up to almost 1-1 extensions, a group factor of $X$. The cases $n>1$ in del-Junco Rudolph's theorem 
cannot arise here since $(X / K)^{n \odot}$, which is never minimal for $n>1$, cannot occur as a factor of the minimal flow $Y$.

In $\S 5$ we prove some preliminary lemmas and in $\S 6$ Theorem 6.1 and some corollaries are proved.

In $\$ 0$ we introduce briefly our general setup and recall basic theorems from the structure theory of minimal sets (see [E1], [B], [GI] and mainly [V1]).

\section{General background}

Throughout this paper $T$ is a fixed but arbitrary discrete group. Discreteness is not as severe a restriction as it sounds, because first, most dynamical notions we deal with do not depend on the topology of $T$ and second, in those cases when the topology is significant, one can use slight modifications to obtain the same results for general topological groups.

We write $\beta T$ for the Stone-Čech compactification of $T . M$ will denote a fixed minimal ideal in $\beta T$. $J$ is the set of idempotents in $M$ and $u$ is a fixed element of $J$. The subset $u M=G$ forms a subgroup of the semi-group $M$.

$\beta T$ acts on every flow $(X, T)$ and the orbit closure of a point $x \in X$ is given by $\bar{o}(x)=\{p x: p \in \beta T\}$. The point $x \in X$ is an almost periodic point iff $\bar{o}(x)$ is minimal iff $\bar{o}(x)=\{p x: p \in M\}$. We make the convention that whenever we choose a base point $x_{0}$ in a minimal flow $X$ then $u x_{0}=x_{0}$. If $(X, T)$ is any flow then the set almost periodic points in $X$ is just $J X=\{v x: x \in J, x \in X\}$; e.g. a point $\left(x, x^{\prime}\right) \in X \times X$ is almost periodic iff $\exists v \in J$ with $v\left(x, x^{\prime}\right)=\left(x, x^{\prime}\right)$.

Whenever $X \stackrel{\varphi}{\rightarrow} Y$ is a homomorphism of flows we denote by $R_{\varphi}$ the subflow of $X \times X$ given by

$$
R_{\varphi}=\left\{\left(x, x^{\prime}\right): \varphi(x)=\varphi\left(x^{\prime}\right)\right\} .
$$

A homomorphism (or extension) $X \stackrel{\varphi}{\rightarrow} Y$ of minimal flows is $B c$ (satisfies the Bronstein condition) if $J R_{\varphi}$ is dense in $R_{\varphi}$.

If $\left(X, x_{0}\right)$ is a pointed minimal flow, (i.e. a minimal flow with a distinguished base point $x_{0}$ ) its Ellis group is the subgroup

$$
\mathscr{G}\left(X, x_{0}\right)=\left\{\alpha \in G: \alpha x_{0}=x_{0}\right\} \quad \text { of } G .
$$

A homomorphism $\left(X, x_{0}\right) \stackrel{\varphi}{\rightarrow}\left(Y, y_{0}\right)$ of minimal flows is proximal iff $\mathscr{G}\left(X, x_{0}\right)=$ $\mathscr{G}\left(Y, y_{0}\right)$.

If $v \in J$ then the sets $\left\{B^{-1} \bar{A} \cap v G: A, B \subset T, v \in \bar{B}\right.$ \} (closure in $\beta T$ ) form a basis for a topology on $v G$ called the $\tau$-topology. For any $v, w \in J$, the groups $v G$ and $w G$ are $\tau$-homeomorphic. The groups of the form $\mathscr{G}\left(X, x_{0}\right)$ are exactly the $\tau$-closed subgroups of $G$. If $F \subset G$ is a $\tau$-closed subgroup of $G$ then

$$
F^{\prime}=\bigcap\{\tau \text {-cls }(O): O \text { a } \tau \text {-nbd of } u \text { in } F\}
$$

is a normal subgroup of $F$ and $F / F^{\prime}$ with the quotient $\tau$-topology is a compact Hausdorff topological group. More generally if $(X, T)$ is a minimal flow, $v \in J$ and $v x=x \in X$ then the map $v G \rightarrow v X, v \alpha \mapsto v \alpha x$ defines a quotient topology on $v X$, also called the $\tau$-topology. A basis for the $\tau$-topology at $x$ is given by the sets

$$
O=[U, B]_{v}=B^{-1} U \cap v X,
$$


where $B \subset T$ is such that $v \in \bar{B} \cap M$ and $U$ is a neighbourhood of $x$ in $X$. If $F \subset G$ is a $\tau$-closed subgroup, $\mathscr{G}\left(X, x_{0}\right) \subset F$ and $x=v \alpha x_{0}$ for $\alpha \in G$ then $\bigcap\{\tau$-cls $(O): O$ is a relative $\tau$-nbd of $x$ in $\left.v \alpha F x_{0}\right\}=v \alpha F^{\prime} x_{0}$.

An extension $Z \stackrel{\rho}{\rightarrow} Y$ of minimal flows is an almost periodic extension if it is distal and $F^{\prime} \subset B$ where $F=\mathscr{G}\left(Y, y_{0}\right), B=\mathscr{G}\left(Z, z_{0}\right), \rho\left(z_{0}\right)=y_{0}$. It is a group extension if in addition $B \triangleleft F$. In the latter case $K=F / B$ is a compact Hausdorff topological group which acts on $(Z, T)$ as a group of automorphisms, with $Z / K \cong Y$.

An extension $X \stackrel{m}{\rightarrow} Y$ of minimal flows is called weakly mixing if the flow $\left(R_{\pi}, T\right)$ is topologically ergodic, i.e. for every two non-empty open subsets $U, V$ in $R_{\pi}, \exists t \in T$ with $t U \cap V \neq \varnothing$. We have the following theorem (see [V1]).

TheOREM. Let $X \stackrel{m}{\rightarrow} Y$ be a $\mathrm{Bc}$ homomorphism of minimal sets then there exists a commutative diagram

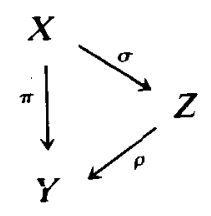

where $\rho$ is almost periodic with $B=F^{\prime} A$. Here $B=\mathscr{G}\left(Z, z_{0}\right), F=\mathscr{G}\left(Y, y_{0}\right)$ and $A=$ $\mathscr{G}\left(X, x_{0}\right) . Z$ is the largest almost periodic extension of $Y$ within $X$ and $\rho$ is trivial (i.e. an isomorphism) iff $\pi$ is weakly mixing.

For the general structure theorems of minimal flows we refer to [V1]. One we use here is the Veech-Ellis theorem which states the identity, for metric flows, of point distal extensions and AI-extensions, i.e. those extensions which can be described as a composition of almost 1-1 extensions, almost periodic extensions and countable inverse limits. The almost 1-1 extensions arise in this theorem via the following construction, called a shadow diagram by Veech.

If $X \stackrel{\rightarrow}{\rightarrow} Z$ is a homomorphism of minimal flows then one can construct a commutative diagram of homomorphisms of minimal flows:

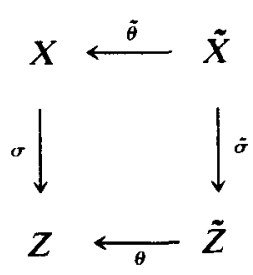

where $\tilde{\sigma}$ is open and $\theta, \tilde{\theta}$ are almost $1-1$. A concrete description using quasifactors (i.e., minimal subsets of $2^{X}$ ) and the circle operation of $\beta T$ on $2^{X}$ is this:

$$
\begin{aligned}
& \tilde{Z}=\left\{p \circ \sigma^{-1}(z): z \in Z, p \in M\right\}, \quad \theta\left(p \circ \sigma^{-1}(z)\right)=p z, \\
& \tilde{X}=\{(x, \tilde{z}): x \in \tilde{z} \in \tilde{Z}\}, \quad \tilde{\theta}(x, \tilde{z})=x,
\end{aligned}
$$

and $\sigma(x, \tilde{z})=\tilde{z}$. For details see, e.g. [Gl]. We say that two minimal flows are almost 1-1 equivalent if they have a common almost $1-1$ extension. We recall that a minimal flow $(X, T)$ is called regular if for any almost periodic point $\left(x, x^{\prime}\right) \in X \times X$ there 
exists $\psi \in$ Aut $(X, T)$ such that $x^{\prime}=\psi(x)$. A homomorphism $X \stackrel{\varphi}{\rightarrow} Y$ of minimal flows is regular if the same holds for every almost periodic point in $\boldsymbol{R}_{\varphi}$.

If $\varphi: X \rightarrow Y$ is a map we also write $\varphi\left(x, x^{\prime}\right)=\left(\varphi(x), \varphi\left(x^{\prime}\right)\right)$ for $\left(x, x^{\prime}\right) \in X \times X$. If $L \subset X \times X$ and $x \in X$ then $L[x]=\left\{x^{\prime} \in X:\left(x, x^{\prime}\right) \in L\right\}$. If also $K \subset X \times X$ then

$$
L \circ K=\left\{\left(x, x^{\prime \prime}\right): \exists x^{\prime} \quad \text { with }\left(x, x^{\prime}\right) \in L,\left(x^{\prime}, x^{\prime \prime}\right) \in K\right\}
$$

With the exceptions $\beta T$ and $M$ all flows are assumed to be metric.

\section{Almost automorphisms and almost simplicity}

Let $(X, T)$ be a minimal flow. A pair $\psi, D$ is an almost automorphism if $D$ is a dense $G_{\delta}$ subset of $X$ which is $T$-invariant and $\psi: D \rightarrow X$ is a continuous 1-1 map such that $\psi \circ t=t \circ \psi \forall t \in T$ and $\psi D$ is also a dense $G_{\delta}$ with $\psi^{-1}: \psi(D) \rightarrow D$ continuous. We sometimes refer only to $\psi$ and write $D_{\psi}$ for $D$.

LEMMA 1.1. Let $(X, T)$ be minimal and $\psi$ an almost automorphism. Then for every $x \in D_{\psi}$ the point $(x, \psi(x))$ is an almost periodic point of $\left(X^{2}, T\right)$, and if $L=\bar{o}(x, \psi(x))$ then $L[x]=\{\psi(x)\}$.

Proof. From $T x \subset D_{\psi}$, the continuity of $\psi$ on $D_{\psi}$ and the relations $t \circ \psi=\psi \circ t \forall t \in T$, we conclude that $\lim t_{i} x=x$ for some net $t_{i} \in T$ implies also $\lim t_{i} \psi(x)=\lim \psi\left(t_{i} x\right)=$ $\psi(x)$. If $v \in J$ is such that $v x=x$ and $\lim t_{i}=v$ in $\beta T$, then also $v \psi(x)=\psi(x)$. This proves that $(x, \psi(x))$ is almost periodic. If $\left(x, x^{\prime}\right) \in L$ then for some sequence $t_{i} \in T$, $\left(x, x^{\prime}\right)=\lim t_{i}(x, \psi(x))=\lim \left(t_{i} x, \psi\left(t_{i} x\right)\right)=(x, \psi(x))$ by continuity of $\psi$. Thus $L[x]=$ $\{\psi(x)\}$.

If $\psi, D$ is an almost automorphism then, let $D_{1}=D \cap \psi(D), D_{2}=D \cap \psi\left(D_{1}\right), \ldots$, $D_{n}=D \cap \psi\left(D_{n-1}\right)$ and $D_{\infty}=\bigcap_{n=1}^{\infty} D_{n}$, then $D_{\infty}=\left\{z \in D: \forall n \exists x \in D, \psi^{n} x=z\right\}$ is a $T$-invariant dense $G_{\delta}$ subset of $X$ and $\psi: D_{\infty} \rightarrow D_{\infty}$ is an automorphism in the usual sense.

Proposition 1.2. Let $(X, T)$ be a minimal flow $\psi$ an almost automorphism. Then there exists a minimal almost 1-1 extension $(\tilde{X}, T) \stackrel{\pi}{\rightarrow}(X, T)$ and an automorphism $\Psi$ of $(\tilde{X}, T)$ such that

(i) $\pi^{-1}(x)=\{\tilde{x}\}$ is a singleton for every $x \in D_{\infty}$.

(ii) $\Psi \tilde{x}=\overline{\psi(x)}, \forall x \in D_{\infty}$.

Proof. Choose $x_{0} \in D_{\infty}$ and let

$$
\tilde{X}=\bigvee_{n \in Z}\left(X, \psi^{n}\left(x_{0}\right)\right):=\bar{o}\left(\ldots, \psi^{-1}\left(x_{0}\right), x_{0}, \psi\left(x_{0}\right), \ldots\right) \subset X^{Z} .
$$

The proof of Lemma 1.1 shows that $\tilde{x}_{0}=\left(\ldots, \psi^{-1}\left(x_{0}\right), x_{0}, \psi\left(x_{0}\right), \ldots\right)$ is an almost periodic point in $\left(X^{Z}, T\right)$ so that $(\tilde{X}, T)$ is minimal. Let $\pi: \tilde{X} \rightarrow X$ be the projection on the zeroth coordinate. If $y_{0} \in D_{\infty}$ and $\tilde{x} \in \tilde{X}$ with $\pi(\tilde{x})=y_{0}$; choose $t_{i} \in T$ with $t_{i} \tilde{x}_{0} \rightarrow \tilde{x}=\left(\ldots y_{-1}, y_{0}, y_{1} \ldots\right)$. By continuity of $\psi$ on $D_{\infty}, \psi^{n}\left(t_{i} x_{0}\right) \rightarrow \psi^{n}\left(y_{0}\right)$ and therefore $y_{n}=\psi^{n}\left(y_{0}\right) \forall n \in \mathbb{Z}$. Thus $\pi^{-1}\left(y_{0}\right)=\{\tilde{x}\}$ and (i) is proved.

Let $S$ be the shift on $X^{Z}$. If we choose $t_{i} \in T$ with $\lim t_{i} x_{0}=\psi\left(x_{0}\right)$ then clearly $\lim t_{i} \tilde{x}_{0}=S \tilde{x}_{0}$. Now $S \circ t=t \circ S(t \in T)$ and the minimality of $\tilde{X}$ imply that $S \tilde{X}=\tilde{X}$. Claim (ii) of the proposition now follows when we define $\Psi=\left.S\right|_{\tilde{X}}$. 
Motivated by $[\mathbf{J}]$ we now introduce

Definition. A metric minimal flow $(X, T)$ is (two fold) almost simple (AS) if there exists a $T$-invariant dense $G_{\delta}$ subset $X^{*} \subset X$ such that for every $x, x^{\prime} \in X^{*}$ either $\bar{o}\left(x, x^{\prime}\right)=X \times X$ or there exists an almost automorphism $\psi$ with $x \in D_{\psi} \subset X^{*}$ such that $\psi(x)=x^{\prime}$. (Since we can replace $D_{\psi}$ by $\psi^{-1}\left(\psi(D) \cap X^{*}\right)$ there is no loss in generality if we assume also that $\psi\left(D_{\psi}\right) \subset X^{*}$.)

Proposition 1.3. Let $(X, T) \stackrel{\pi}{\rightarrow}(Y, T)$ be an almost 1-1 homomorphism of metric minimal flows; $(X, T)$ is $A S$ iff $(Y, T)$ is AS.

Proof. Let $Y_{0}=\left\{y \in Y: \pi^{-1}(y)\right.$ is a singleton. $\}, X_{0}=\pi^{-1}\left(Y_{0}\right)$. Then $Y_{0}$ and $X_{0}$ are $T$-invariant dense $G_{\delta}$ subsets of $Y$ and $X$ respectively, and $\pi: X_{0} \rightarrow Y_{0}$ is a homeomorphism.

Suppose $Y$ is an AS flow with $Y^{*} \subset Y$ as the required subset. Put $X^{*}=$ $\pi^{-1}\left(Y^{*} \cap Y_{0}\right)$; then $X^{*}$ is clearly $T$-invariant dense $G_{\delta}$. Let $x, x^{\prime} \in X^{*}$ and let $y=\pi(x), y^{\prime}=\pi\left(x^{\prime}\right)$. If $\bar{o}\left(y, y^{\prime}\right)=Y \times Y$ then in particular $\bar{o}\left(y, y^{\prime}\right) \supset Y_{0} \times Y_{0}$. Thus $\bar{o}\left(x, x^{\prime}\right) \supset X_{0} \times X_{0}$ and since the latter is dense in $X \times X$ we have $\bar{o}\left(x, x^{\prime}\right)=X \times X$. If on the other hand $\bar{o}\left(y, y^{\prime}\right) \neq Y \times Y$ then by almost simplicity of $Y$ there exists an almost automorphism $\psi$ with $D_{\psi} \subset Y^{*}$ and $\psi(y)=y^{\prime}$. Without loss of generality we can assume that $D_{\psi}, \psi\left(D_{\psi}\right) \subset Y^{*} \cap Y_{0}$. Then $\pi^{-1} \circ \psi \circ \pi$ restricted to $\pi^{-1}\left(D_{\psi}\right)$ is an almost automorphism $\varphi$ of $(X, T)$ with $D_{\varphi}=\pi^{-1}\left(D_{\psi}\right) \subset X^{*}$ and $\varphi(x)=x^{\prime}$.

Conversely assume $(X, T)$ is AS with $X^{*}$ as the required set. Put $Y^{*}=\pi\left(X^{*} \cap X_{0}\right)$ and it is now easy to see that with respect to $Y^{*},(Y, T)$ is AS.

Problem. Is every AS flow almost 1-1 equivalent to a regular flow?

We say that a metric minimal flow $(X, T)$ has (two fold) almost minimal self-joinings (AMSJ) if there exists a $T$-invariant dense $G_{\delta}$ subset $X^{*}$ of $X$ such that for all pairs $x, x^{\prime} \in X^{*}$ either $\bar{o}\left(x, x^{\prime}\right)=X \times X$ or $x^{\prime}=t x$ for some $t \in T$. Again it is easy to see that AMSJ is an invariant of the almost 1-1 equivalence relation. Clearly every AMSJ flow is AS when $T$ is commutative. We assume this is the next.

Proposition 1.4. Let $(X, T)$ be a metric minimal weakly mixing flow, $(X, T) \stackrel{\pi}{\rightarrow}(Y, T)$ a group extension and suppose that $(Y, T)$ has AMSJ; then $(X, T)$ is AS.

Proof. Let $Y^{*} \subset Y$ be the subset ensuring AMSJ for $(Y, T)$. Let $K$ be the compact group of automorphisms of $(X, T)$ such that $\pi: X / K \cong Y$. Let $X^{*}=\pi^{-1}\left(Y^{*}\right)$, then $X^{*}$ is a $T$-invariant dense $G_{\delta}$ subset of $X$. Let $x, x^{\prime} \in X^{*}$ and denote $L=\bar{o}\left(x, x^{\prime}\right)$. If $\pi(L) \neq Y \times Y$ then by AMSJ of $Y$, there exists $t \in T$ such that $t \pi(x)=\pi\left(x^{\prime}\right)$ so that $k t x=x^{\prime}$ for some $k \in K$ and the AS requirement is satisfied with the global automorphism $k t$. If $\pi(L)=Y \times Y$ then

$$
X \times X=\bigcup\left\{\left(k \times k^{\prime}\right) L:\left(k, k^{\prime}\right) \in K \times K\right\} .
$$

Since $X$ is weakly mixing there exists $\left(z, z^{\prime}\right) \in X \times X$ with $\bar{o}\left(z, z^{\prime}\right)=X \times X$. If $\left(z, z^{\prime}\right) \in\left(k \times k^{\prime}\right) L$ then $\left(k^{-1} z, k^{\prime-1} z^{\prime}\right)$ is a point of $L$ whose orbit is dense in $X \times X$. Thus $L=X \times X$. 
Since the Chacon minimal set has AMSJ, $[\mathbf{J}]$, it follows that every minimal metric flow which is almost 1-1 equivalent to a weakly mixing group extension of the Chacon minimal set is AS.

In the proof of the next lemma we use an idea of N. Markley [J, Proposition 3.11]. This will be used repeatedly throughout the paper.

A map $X \stackrel{\oplus}{\rightarrow} Y$ is called semi-open if int $\varphi(V) \neq \varnothing$ for every $V \subset X$ open and non-empty.

LeMma 1.5. Let $(X, T)$ be a minimal flow, $X^{*}$ a dense $G_{\delta}$ subset of $X$ and $W \subset X^{2}$ a closed invariant subset. If the projection maps $\pi_{j}: W \rightarrow X(j=1,2)$ are semi-open then $W^{*}=W \cap\left(X^{*} \times X^{*}\right)$ is dense $G_{\delta}$ in $W$.

Proof. Let $V \subset W$ be a non-empty open subset. By assumption int $\pi_{j}(V) \neq \varnothing$, whence int $\pi_{j}(V) \cap X^{*} \neq \varnothing$. Thus $\pi_{j}^{-1}\left(X^{*}\right)$ is a dense $G_{\delta}$ subset of $W(j=1,2)$, and therefore so is $\pi_{1}^{-1}\left(X^{*}\right) \cap \pi_{2}^{-1}\left(X^{*}\right)=W^{*}$.

As observed by J. Auslander, when $X$ is minimal every homomorphism $X \stackrel{\varphi}{\rightarrow} Y$ is semi-open.

LEMMA 1.6. Let $(X, T)$ be an AS flow $X \stackrel{\varphi}{\rightarrow} Y$ an open homomorphism where $Y$ is not a point. Then the set of almost periodic points in $R_{\varphi}$ contains a dense $G_{\delta}$ subset. In particular $\varphi$ is a $\mathrm{Bc}-e x t e n s i o n$.

Proof. Let $\pi_{j}: R_{\varphi} \rightarrow X(j=1,2)$ be the projections on $X$. It is easy to check that these also are open maps.

By Lemma $1.5 R_{\varphi}^{*}=R_{\varphi} \cap\left(X^{*} \times X^{*}\right)$ is dense $G_{\delta}$ in $R_{\varphi}$. But if $\left(x, x^{\prime}\right) \in R_{\varphi}^{*}$ then $\bar{o}\left(x, x^{\prime}\right) \subset R_{\varphi} \neq X \times X$ and by the AS property $x^{\prime}=\psi(x)$ for some almost automorphic $\psi$. By Lemma $1.1\left(x, x^{\prime}\right)$ is almost periodic.

Proposition 1.7. Let $(X, T)$ be an AS flow $X \stackrel{\oplus}{\rightarrow} Y$ a homomorphism with non-trivial $Y$. Then $\varphi$ is an AI hence also a point distal extension.

Proof. Let $X_{\infty} \stackrel{\varphi_{\infty}}{\rightarrow} Y_{\infty}$ be the canonical AI tower corresponding to $\varphi$ then $X_{\infty}$ as an almost 1-1 extension of $X$ is also AS and the extension $\varphi_{\infty}$ is open. By Lemma 1.6 it is also a Bc-extension.

By maximality of $Y_{\infty}$ it follows that $\varphi_{\infty}$ is weakly mixing (see [V1, Theorem 2.6.4]). Since both the set of points with dense orbit and the set $\left(X^{*} \times X^{*}\right) \cap R_{\varphi_{\infty}}$ are dense $G_{\delta}$ in $R_{\varphi_{\infty}}$, we conclude that $R_{\varphi_{\infty}}$ is minimal. Thus $R_{\varphi_{\infty}}=\Delta_{X_{\infty}}, \varphi_{\infty}$ is $1-1$, and $Y_{\infty}=X_{\infty}$ is a strictly AI extension of $Y$. Since $X_{\infty}$ is an almost $1-1$ extension of $X$ it follows that $\varphi$ is AI.

COROLlaRY 1.8. If $(X, T)$ is AS and purely weakly mixing ([E-G]), then $(X, T)$ is essentially prime; i.e., every factor map of $(X, T)$ is almost 1-1.

Proof. Suppose $X \stackrel{\varphi}{\rightarrow} Y$ is a factor. By Proposition $1.7 \varphi$ is a point distal homomorphism and by definition of pure weak mixing $\varphi$ is proximal hence almost 1-1.

Proposition 1.9. If $(X, T)$ is $\mathrm{AS}$ and $X \stackrel{\varphi}{\rightarrow} Y$ is an almost periodic extension then $\varphi$ is a group extension. 
Proof. By [E1] there exists a commutative diagram

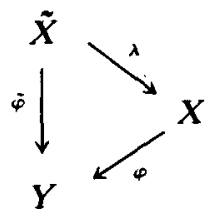

such that $\tilde{\varphi}$ is a group extension, say $\tilde{\varphi}: \tilde{X} \rightarrow \tilde{X} / K \cong Y$ where $K$ is a compact group of automorphisms of $(\tilde{X}, T)$. Let $K_{0}=\left\{k \in K: \lambda\left(\tilde{x}_{0} k\right)=\lambda\left(\tilde{x}_{0}\right)=x_{0}\right\}$ then $\lambda$ is not 1-1 iff $K_{0}$ is a non-normal subgroup of $K$. Assume $k^{-1} K_{0} k \neq K_{0}$ for some $k \in K$ and choose $k_{0} \in K_{0}$ with $k^{-1} k_{0} k \notin K_{0}$.

Let $L=\bar{o}\left(x_{0}, x_{1}\right)=\bar{o}\left(\lambda\left(\tilde{x}_{0}\right), \lambda\left(\tilde{x}_{0} k\right)\right)$. Choose a sequence $t_{i} \in T$ such that $\lim t_{i} \tilde{x}_{0}=$ $\tilde{x}_{0} k_{0}$, then

$$
\begin{aligned}
\lim t_{i}\left(x_{0}, x_{1}\right) & =\lim \lambda\left(t_{i} \tilde{x}_{0}, t_{i} \tilde{x}_{0} k\right)=\lambda\left(\tilde{x}_{0} k_{0}, \tilde{x}_{0} k_{0} k\right) \\
& =\left(x_{0}, x_{2}\right),
\end{aligned}
$$

where $x_{2}=\lambda\left(\tilde{x}_{0} k_{0} k\right)=\lambda\left(\tilde{x}_{0} k\left(k^{-1} k_{0} k\right)\right) \neq \lambda\left(\tilde{x}_{0} k\right)=x_{1}$. Since equality will imply by equivariance of $\lambda$ that also $\lambda\left(\tilde{x}_{0}\right)=\lambda\left(\tilde{x}_{0} k^{-1} k_{0} k\right)$, i.e., $k^{-1} k_{0} k \in K_{0}$, a contradiction.

By Lemma $1.5 L^{*}=L \cap\left(X^{*} \times X^{*}\right)$ is dense $G_{\delta}$ in $L$. Choose $\left(x, x^{\prime}\right) \in L^{*}$; by the AS property $x^{\prime}=\psi(x)$ for an almost automorphism $\psi$. Moreover, if $\left(x, x^{\prime \prime}\right) \in L$ then there exists a sequence $t_{i} \in T$ with $\lim t_{i}\left(x, x^{\prime}\right)=\left(x, x^{\prime \prime}\right)$. But $t_{i} x \in X^{*}$ and $t_{i} x \rightarrow x$ imply

$$
\lim t_{i}\left(x, x^{\prime}\right)=\lim \left(t_{i} x, \psi\left(t_{i} x\right)\right)=(x, \psi(x))=\left(x, x^{\prime}\right)
$$

so that $x^{\prime}=x^{\prime \prime}$.

Finally choose a sequence $t_{i} \in T$ with $\lim t_{i} x_{0}=x$ then for $j=1,2$ we have

$$
\lim _{i} t_{i}\left(x_{0}, x_{j}\right)=\left(x, \lim _{i} t_{i} x_{j}\right) \in L
$$

So that $\lim _{i} t_{i} x_{1}=\lim _{i} t_{i} x_{2}$, i.e. $x_{1}$ and $x_{2}$ are proximal. Since $L \subset R_{\varphi}$ and $\varphi$ is a distal extension this is impossible. Thus $K_{0} \triangleleft K$ hence $K_{0}=e$ and $\varphi$ is a group extension.

As an application of Proposition 1.2 here is an alternative proof for Proposition 1.9. Let $A=\mathscr{G}\left(X, x_{0}\right), F=\mathscr{G}\left(Y, y_{0}\right)$ where $y_{0}=\varphi\left(x_{0}\right)$. It suffices to show that $A \triangleleft F$. Let $\gamma \in F$ then $\bar{o}\left(x_{0}, \gamma x_{0}\right)=N$ is minimal. Hence $N^{*}=N \cap\left(X^{*} \cap X^{*}\right)$ is a dense $G_{\delta}$ subset of $N$ (Lemma 1.5) and if $\left(x, x^{\prime}\right) \in N^{*}$ then by AS, $x^{\prime}=\psi(x)$ for an almost automorphism $\psi$. By Proposition 1.2 there exists an almost 1-1 extension $\tilde{X} \stackrel{\theta}{\rightarrow} X$ and an automorphism $\Psi$ of $(\tilde{X}, T)$ extending $\theta^{-1} \circ \psi \circ \theta$ on $D_{\infty} \subset D_{\psi} \cap X_{0} \subset X^{*}$ where $X_{0}=\left\{x \in X: \theta^{-1}(x)\right.$ is a singleton $\}$. If $\Gamma_{\Psi}=\{(\tilde{x}, \Psi(\tilde{x})): \tilde{x} \in \tilde{X}\}$ then clearly $\theta\left(\Gamma_{\Psi}\right)=N$. Now $\bar{o}\left(\tilde{x}_{0}, \gamma \tilde{x}_{0}\right)$ is also a minimal set with $\theta\left(\bar{o}\left(\tilde{x}_{0}, \gamma \tilde{x}_{0}\right)\right)=N$. Thus $\theta^{-1}\left(x, x^{\prime}\right)=\left(\tilde{x}, \tilde{x}^{\prime}\right) \in \Gamma_{\Psi} \cap \bar{o}\left(\tilde{x}_{0}, \gamma \tilde{x}_{0}\right)$ whence $\Gamma_{\Psi}=\bar{o}\left(\tilde{x}_{0}, \gamma \tilde{x}_{0}\right)$. In particular $\gamma \tilde{x}_{0}=$ $\Psi\left(\tilde{x}_{0}\right)$. Since $\theta$ is proximal $\mathscr{G}\left(\tilde{X}, \tilde{x}_{0}\right)=\mathscr{G}\left(X, x_{0}\right)=A$ and for $\alpha \in A$

$$
\alpha \gamma \tilde{x}_{0}=\alpha \Psi\left(\tilde{x}_{0}\right)=\Psi\left(\alpha \tilde{x}_{0}\right)=\Psi\left(\tilde{x}_{0}\right)=\gamma \tilde{x}_{0}
$$

so that $\gamma^{-1} \alpha \gamma \tilde{x}_{0}=\tilde{x}_{0}$ and $\gamma^{-1} \alpha \gamma \in A$.

Proposition 1.10. Let $(X, T) \stackrel{\pi}{\rightarrow}(Y, T)$ be a regular, point distal homomorphism of minimal flows. Then for every Y-distal point $x \in X, \pi^{-1}(y)=A x$ where $y=\pi(x)$ and 
$A=\{\psi \in$ Aut $(X, T): \pi \circ \psi=\pi\}$. In particular if $(X, T)$ is a regular point distal flow then Aut $(X, T)$ is transitive on $X$ and $(X, T)$ is distal (hence also equicontinuous by Proposition 3.1 when $X$ is metrizable).

Proof. Let $\pi\left(x^{\prime}\right)=y$ and choose $v \in J$ with $v x^{\prime}=x^{\prime}$. Then $v y=y$ and since $x$ is $Y$-distal $v x=x$, so that $\left(x, x^{\prime}\right)$ is an almost periodic point of $R_{\pi}$. The regularity of $\pi$ implies that $x^{\prime}=\psi(x)$ for some $\psi \in A u t(X, T)$ and necessarily $\psi \in A$.

\section{The existence of saturated orbit closures}

LEMMA 2.1. Let $X \stackrel{\varphi}{\rightarrow} Y$ be an open homomorphism of metric flows. Let $\mathscr{V}=\{V \subset X: V$ open and $\varphi(V)=Y\}$. Then there exists a countable sub-collection $\left\{V_{i}\right\}_{i=1}^{\infty}$ of $\mathscr{V}$ such that every element of $\mathcal{V}$ contains some $V_{i}$.

Proof. Let $V \in \mathscr{V}$ and for $\varepsilon>0$ denote $V_{\varepsilon}=\left\{x \in V: d\left(x, V^{c}\right) \geq \varepsilon\right\}$. If for every $n$, $\varphi\left(V_{1 / n}\right) \neq Y$, then there exist $y_{n} \in Y \backslash \varphi\left(V_{1 / n}\right)$. Let $y_{n_{k}} \rightarrow y$; by assumption there exists $x \in V$ with $\varphi(x)=y$. Let $\delta=d\left(x, V^{c}\right)$. Since $\varphi$ is open we can find $x_{n_{k}} \in X$ with $\varphi\left(x_{n_{k}}\right)=y_{n_{k}}$ such that $x_{n_{k}} \rightarrow x$. But then $x_{n_{k}}$ is eventually in $V_{\delta / 2}$ and $y_{n_{k}} \in \varphi\left(V_{\delta / 2}\right)$ a contradiction. We proved: for every $V \in \mathscr{V}$ there exists a closed subset $L \subset V$ with $\varphi(L)=Y$. Let $U=\left\{U_{i}\right\}_{i=1}^{\infty}$ be a countable basis for open sets on $X$, then a finite subset $\left\{U_{i_{1}}, \ldots, U_{i_{k}}\right\}$ covers $L$ and satisfies $\bigcup_{j=1}^{k} U_{i_{j}} \subset V$. Thus

$$
\mathscr{V}_{0}=\left\{V=\bigcup_{j=1}^{k} U_{i_{j}}: U_{i_{j}} \in \mathcal{U} \quad \text { and } \varphi(V)=Y\right\}
$$

is the required countable sub-collection.

LEMMA 2.2. If

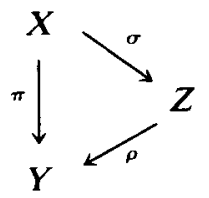

is a commutative diagram and $\sigma$ is open then $\sigma: R_{\pi} \rightarrow R_{\rho}$ is also open.

Proof. Let $R_{\rho} \ni\left(z_{i}, z_{i}^{\prime}\right) \rightarrow\left(z, z^{\prime}\right)$ and $\left(x, x^{\prime}\right) \in R_{\pi}$ with $\sigma\left(x, x^{\prime}\right)=\left(z, z^{\prime}\right)$. By openness of $\sigma$ there exist sequences $x_{i}, x_{i}^{\prime} \in X$ such that $\sigma\left(x_{i}\right)=z_{i}, \sigma\left(x_{i}^{\prime}\right)=z_{i}^{\prime}$ and $x_{i} \rightarrow x, x_{i}^{\prime} \rightarrow x^{\prime}$. Clearly then $R_{\pi} \ni\left(x_{i}, x_{i}^{\prime}\right) \rightarrow\left(x, x^{\prime}\right)$ and $\sigma\left(x, x^{\prime}\right)=\left(z, z^{\prime}\right)$ so that $\sigma: R_{\pi} \rightarrow R_{p}$ is open.

LEMMA 2.3. Let $X \stackrel{\pi}{\rightarrow} Y$ be a homomorphism of minimal flows. Let $F=\mathscr{G}\left(Y, y_{0}\right)$ and $A=\mathscr{G}\left(X, x_{0}\right)$ with $y_{0}=\pi\left(x_{0}\right)$. Let $\left(x, x^{\prime}\right)=\left(v \alpha x_{0}, v \beta x_{0}\right)$ where $\alpha, \beta \in G$ and $v \in J$, be an almost periodic point in $\boldsymbol{R}_{\pi}$. Then for every neighbourhood $V$ of $\left(x, x^{\prime}\right)$ in $\boldsymbol{R}_{\pi}$ we have

$$
\left(v \alpha F^{\prime} x_{0}\right) \times\left(v \beta F^{\prime} x_{0}\right) \subset \overline{T\left(V \cap J R_{\pi}\right)} \text {. }
$$

Proof. Let $U, U^{\prime}$ be open subsets of $X$ such that $\left(x, x^{\prime}\right) \in\left(U \times U^{\prime}\right) \cap J R_{\pi} \subset V$. Since $v\left(x, x^{\prime}\right)=\left(x, x^{\prime}\right)$ there exists a subset $B \subset T$ such that $v \in \bar{B} \cap M$ (closure in $\beta T$ ) and $B x^{\prime} \subset U^{\prime}$.

The set $O=[U, B]_{v}=B^{-1} U \cap v \pi^{-1} \pi(x)$ is a $\tau$-neighbourhood of $x$ in $v \pi^{-1} \pi(x)=$ $v \alpha F x_{0}$. 
If $x_{1} \in O$, there exists $t \in B$ with $x_{2}=t x_{1} \in U$. Since $\pi\left(x_{1}\right)=\pi(x)=\pi\left(x^{\prime}\right)$ we have

$$
\left(x_{1}, x^{\prime}\right)=t^{-1}\left(x_{2}, t x^{\prime}\right) \in t^{-1}\left(U \times B x^{\prime}\right) \cap J R_{\pi} \subset T\left(\left(U \times U^{\prime}\right) \cap J R_{\pi}\right) .
$$

Thus $O \times\left\{x^{\prime}\right\} \subset T\left(\left(U \times U^{\prime}\right) \cap J R_{n}\right)$ and therefore

$$
\tau-\operatorname{cls}\left(O \times\left\{x^{\prime}\right\}\right) \subset \overline{T\left(\left(U \times U^{\prime}\right) \cap J R_{\pi}\right)} .
$$

Since $O$ is an arbitrary basic $\tau$-neighbourhood of $x$ in $v \alpha F x_{0}$ we conclude that

$$
\bigcap_{o} \tau-\operatorname{cls}\left(O \times\left\{x^{\prime}\right\}\right)=\left(v \alpha F^{\prime} x_{0}\right) \times\left\{x^{\prime}\right\} \subset \overline{T\left(V \cap J R_{\pi}\right)} .
$$

Now $\left(x, x^{\prime}\right) \in R_{\pi}$ implies $\alpha^{-1} \beta \in F$ and $F^{\prime} \triangleleft F$. Thus for every $\gamma, \delta \in F^{\prime}, \alpha^{-1} \beta \delta=$ $\delta^{\prime} \alpha^{-1} \beta$ for $\delta^{\prime} \in F^{\prime}$ and

$$
\begin{aligned}
\left(v \alpha \gamma x_{0}, v \beta \delta x_{0}\right) & =v \alpha\left(\gamma x_{0}, \alpha^{-1} \beta \delta x_{0}\right) \\
& =v \alpha\left(\gamma x_{0}, \delta^{\prime} \alpha^{-1} \beta x_{0}\right) \\
& =v \alpha \delta^{\prime}\left(\delta^{\prime-1} \gamma x_{0}, \alpha^{-1} \beta x_{0}\right) \\
& =v \alpha \delta^{\prime} \alpha^{-1}\left(\alpha \delta^{\prime-1} \gamma x_{0}, \beta x_{0}\right) \\
& \in\left(v \alpha \delta^{\prime} \alpha^{-1}\right)\left[\left(v \alpha F^{\prime} x_{0}\right) \times\left\{x^{\prime}\right\}\right] \\
& \subset \overline{T\left(V \cap J R_{\pi}\right)},
\end{aligned}
$$

as claimed.

LeMMA 2.4. Let

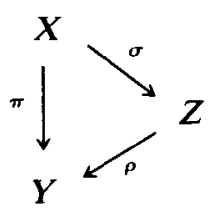

be a commutative diagram of minimal flows such that $\mathscr{G}\left(Z, z_{0}\right)=F^{\prime} A$ where $F=$ $\mathscr{G}\left(Y, y_{0}\right), A=\mathscr{G}\left(X, x_{0}\right)$. Then for a non-empty relatively open $U \subset \overline{J R_{\pi}}$ such that $\sigma(U)=R_{\rho}$ we have $\overline{T U}=\overline{J R_{\pi}}$.

Proof. Let $W=\left(U_{1} \times U_{2}\right) \cap \overline{J R_{\pi}} \subset U$ where $U_{i}(i=1,2)$ are open subsets of $X$. Let $\left(x_{1}, x_{2}\right) \in W$ be an almost periodic point say $\left.v\left(x_{1}, x_{2}\right)\right)=\left(x_{1}, x_{2}\right)$ for $v \in J$. By Lemma $2.3 \overline{T W} \supset\left(v \alpha F^{\prime} x_{0}\right) \times\left(v \beta F^{\prime} x_{0}\right)=\left(v \sigma^{-1} \sigma\left(x_{1}\right)\right) \times\left(v \sigma^{-1} \sigma\left(x_{2}\right)\right)$, where $x_{1}=v \alpha x_{0}, x_{2}=$ $v \beta x_{0}$. For any $\left(x, x^{\prime}\right) \in W$ choose $\left(x_{i}, x_{i}^{\prime}\right) \in W \cap J R_{\pi}$ say $w_{i}\left(x_{i}, x_{i}^{\prime}\right)=\left(x_{i}, x_{i}^{\prime}\right)$ such that $\left(x_{i}, x_{i}^{\prime}\right) \rightarrow\left(x, x^{\prime}\right)$. We can find $\alpha_{i}, \alpha, \alpha_{i}^{\prime}, \alpha^{\prime} \in G, w_{i}, w, w^{\prime} \in J$ such that $w_{i} \alpha_{i} \rightarrow w \alpha$, $w_{i} \alpha_{i}^{\prime} \rightarrow w^{\prime} \alpha^{\prime}$ and $w_{i} \alpha_{i} x_{0}=x_{i}, w_{i} \alpha_{i}^{\prime} x_{0}=x_{i}^{\prime}, w \alpha x_{0}=x, w^{\prime} \alpha^{\prime} x_{0}=x^{\prime}$. If $\gamma, \gamma^{\prime} \in F^{\prime}$ then

$$
\overline{T W} \ni\left(w_{i} \alpha_{i} \gamma x_{0}, w_{i} \alpha_{i}^{\prime} \gamma^{\prime} x_{0}\right) \rightarrow\left(w \alpha \gamma x_{0}, w^{\prime} \alpha^{\prime} \gamma^{\prime} x_{0}\right)
$$

so that also $\overline{T U} \supset \overline{T W} \supset\left(w \sigma^{-1} \sigma(x)\right) \times\left(w^{\prime} \sigma^{-1} \sigma\left(x^{\prime}\right)\right)$.

Now let $\left(\bar{x}, \bar{x}^{\prime}\right) \in J R_{\pi}$; by assumption there is a point $\left(x, x^{\prime}\right) \in U$ with $\sigma\left(x, x^{\prime}\right)=$ $\sigma\left(\bar{x}, \bar{x}^{\prime}\right)=\left(z, z^{\prime}\right) \in R_{\rho}$. If $w x=x, w^{\prime} x^{\prime}=x^{\prime}$ for $w, w^{\prime} \in J$ then by the above $\overline{T U} \supset$ $\left(w \sigma^{-1}(z)\right) \times\left(w^{\prime} \sigma^{-1}\left(z^{\prime}\right)\right)$ hence also $\overline{T U} \supset\left(v \sigma^{-1}(z)\right) \times\left(v \sigma^{-1}\left(z^{\prime}\right)\right) \ni\left(\bar{x}, \bar{x}^{\prime}\right)$ where $v\left(\bar{x}, \bar{x}^{\prime}\right)=\left(\bar{x}, \bar{x}^{\prime}\right), v \in J$. Thus $\overline{T U} \supset J R_{\pi}$ and finally $\overline{T U} \supset \overline{J R_{\pi}}$. 
Proposition 2.5. Let

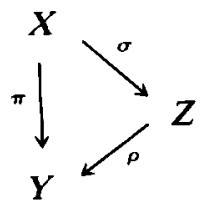

be a commutative diagram of homomorphisms of minimal metric flows such that $\pi$ is open Bc-extension and $\rho$ is the maximal a.p. extension of $Z$ inside $X$. Let

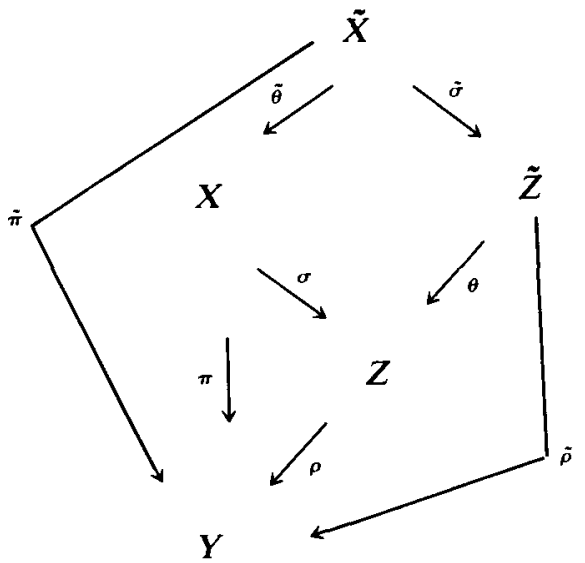

be the given diagram with a Veech shadow diagram for $\sigma$ attached. Thus $\theta$ and $\tilde{\theta}$ are almost 1-1 and $\tilde{\sigma}$ is open. Suppose further that $\tilde{\pi}$ happens to be a Bc-extension. Then there exists a dense $G_{\delta}$ subset $S$ of $R_{\tilde{\pi}}$ such that $\left(\tilde{x}, \tilde{x}^{\prime}\right) \in S$ implies $R_{\tilde{\sigma}} \circ \bar{o}\left(\tilde{x}, \tilde{x}^{\prime}\right) \circ R_{\tilde{\sigma}}=$ $\bar{o}\left(\tilde{x}, \tilde{x}^{\prime}\right)$, i.e. the orbit closure of $\left(\tilde{x}, \tilde{x}^{\prime}\right)$ is $R_{\tilde{\sigma}}$ saturated.

Proof. Applying Lemma 2.2 to the diagram

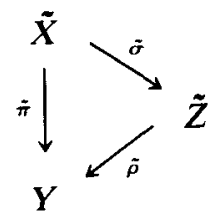

we see that $\tilde{\sigma}: R_{\dot{m}} \rightarrow R_{\tilde{\rho}}$ is an open homomorphism. From Lemma 2.1 we now deduce the existence of a sequence $\left\{V_{i}\right\}_{i=1}^{\infty}$ of open subsets of $R_{\tilde{\pi}}$ such that for each $i V_{i} \in \mathscr{V}$ and for every $V \in \mathscr{V}$ there exists an $i$ with $V_{i} \subset V$. Here $\mathscr{V}=\left\{V: V \subset R_{\tilde{\pi}}\right.$ is open and $\left.\tilde{\sigma}(V)=R_{\dot{\rho}}\right\}$. Our assumptions that $\pi$ is $\mathrm{Bc}$ and that $\rho$ is the maximal a.p. extension of $Y$ inside $X$ imply that $F^{\prime} A=\mathscr{G}\left(Z, z_{0}\right)$ where $F=\mathscr{G}\left(Y, y_{0}\right), A=\mathscr{G}\left(X, x_{0}\right)$, [E1]. Since $\theta$ and $\tilde{\theta}$ are almost $1-1$, hence proximal, we also have $A=\mathscr{G}\left(\tilde{X}, \tilde{x}_{0}\right)$ and $F^{\prime} A=\mathscr{G}\left(\tilde{Z}, \tilde{z_{0}}\right)$. Thus we can apply Lemma 2.4 to the diagram

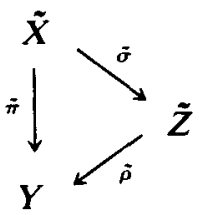

and conclude that $\overline{T U}=\overline{J R_{\tilde{\pi}}}$ for every relatively open $U \subset \overline{J R_{\tilde{\pi}}}$ with $\tilde{\sigma}(U)=R_{\tilde{\rho}}$. 
Since we also assumed that $\tilde{\pi}$ is $\mathrm{Bc}, \overline{J R_{\tilde{\pi}}}=R_{\tilde{\pi}}$ and $\overline{T V}=R_{\dot{\pi}}$ for every $V \in \mathscr{V}$. Baire's theorem now shows that $S^{\prime}=\bigcap_{i=1}^{\infty} T V_{i}$ is a dense $G_{\delta}$ subset of $R_{\tilde{\pi}}$. It also follows that $\overline{J R}_{\tilde{\rho}}=R_{\tilde{\rho}}$ and hence the projections of $R_{\tilde{\rho}}$ on $\tilde{Z}$ are semiopen. Let $Z_{0}=\left\{z \in Z:\left|\theta^{-1}(z)\right|=1\right\}, \tilde{Z}_{0}=\theta^{-1}\left(Z_{0}\right)$.

Since $R_{\rho}$ consists of almost periodic points so does $R_{\tilde{\rho}} \cap \tilde{Z}_{0} \times \tilde{Z}_{0}$ which by Lemma 1.5 is dense $G_{\delta}$ in $R_{\tilde{\rho}}$. Put $S=S^{\prime} \cap \tilde{\sigma}^{-1}\left(R_{\tilde{\rho}} \cap \tilde{Z}_{0} \times \tilde{Z}_{0}\right)$. Let $\left(\tilde{x}, \tilde{x}^{\prime}\right) \in S$ and denote $L=\bar{o}\left(\tilde{x}, \tilde{x}^{\prime}\right)$. If there exists $\left(\tilde{z}_{1}, \tilde{z}_{2}\right) \in R_{\tilde{\rho}}$ with $\tilde{\sigma}^{-1}\left(\tilde{z}_{1}\right) \times \tilde{\sigma}^{-1}\left(\tilde{z}_{2}\right) \subset L$ then by minimality of $\tilde{\sigma}(L)$ and openness of $\tilde{\sigma}$

$$
R_{\tilde{\sigma}} \circ L \circ R_{\tilde{\sigma}}=L
$$

and we are done. Otherwise, $V=R_{\tilde{\pi}} \backslash L$ is an element of $\mathscr{V}$ and choosing $i$ with $V_{i} \subset V$ we get a contradiction since by definition of $S$ there exists $t \in T$ with $t\left(\tilde{x}, \tilde{x}^{\prime}\right) \in V_{i} \subset V$.

\section{Two special cases}

Proposition 3.1. Let $(X, T)$ be a minimal metric flow and let $X \stackrel{\pi}{\rightarrow} Y$ be a regular, distal homomorphism, then $\pi$ is a group extension. In particular if $(X, T)$ is a minimal regular metric distal flow then $(X, T)$ is equicontinuous.

Proof. Let $Z \stackrel{\rho}{\rightarrow} Y$ be the maximal almost periodic extension of $Y$ within $X$ so that we have the commutative diagram

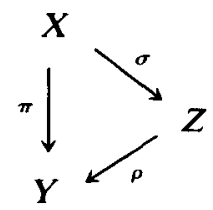

as in Proposition 2.5. Now $\sigma$ as a distal homomorphism is open so that the shadow diagram in Proposition 2.5 degenerates $(\tilde{Z}=Z, \tilde{X}=X, \tilde{\pi}=\pi, \tilde{\sigma}=\sigma)$ and we conclude that there exists $\left(x, x^{\prime}\right) \in R_{\pi}$ with

$$
R_{\sigma} \circ \bar{o}\left(x, x^{\prime}\right) \circ R_{\sigma}=\bar{o}\left(x, x^{\prime}\right) .
$$

However, since $\pi$ is distal $\left(x, x^{\prime}\right)$ is an almost periodic point and by regularity of $\pi, \bar{o}\left(x, x^{\prime}\right)=\Gamma_{\psi}$ for $\psi \in$ Aut $(X, T)$. Thus $\sigma$ is $1-1$ and $\pi$ is almost periodic. It is easy to see that an almost periodic regular extension is necessarily a group extension.

Proposition 3.2. Let $(X, T)$ be an AS flow and suppose that $(X, T)$ admits a non trivial equicontinuous factor (e.g. this is the case when $(X, T)$ is incontractible [GI] and not weakly mixing). Then $X$ is an almost 1-1 extension of an equicontinuous flow. Proof. Let $X \stackrel{\sigma}{\rightarrow} Z$ be the non trivial equicontinuous factor. By Proposition $1.7 \sigma$ is point distal. Thus $X$ itself is a point distal flow. This implies that the almost periodic points are dense in $X \times X$. Taking $Y$ to be the one point trivial flow we have a diagram

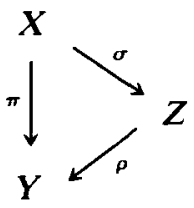


satisfying the hypothesis of Proposition 2.5. Also when we attach the shadow diagram of $\sigma$ to obtain

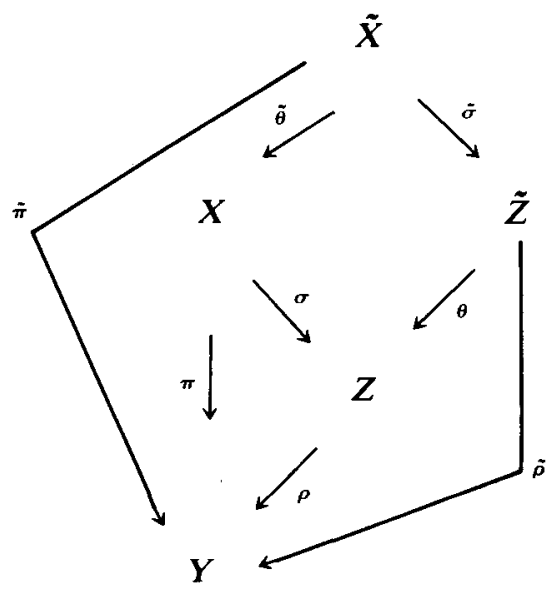

where $\tilde{\sigma}$ is open and $\theta, \tilde{\theta}$ almost $1-1$, then $\tilde{X}$ is also point distal and therefore $\tilde{\pi}$ is Bc and obviously open. Thus all the assumptions in Proposition 2.5 are satisfied and we conclude that there exists a dense $G_{\delta}$ subset $S \subset R_{\tilde{\pi}}=\tilde{X} \times \tilde{X}$ such that $\left(\tilde{x}, \tilde{x}^{\prime}\right) \in S$ implies

$$
R_{\tilde{\sigma}} \circ \bar{o}\left(\tilde{x}, \tilde{x}^{\prime}\right) \circ R_{\tilde{\alpha}}=\overline{\boldsymbol{o}}\left(\tilde{x}, \tilde{x}^{\prime}\right)
$$

Since $\tilde{X}$ is an almost 1-1 extension of $X$ it is AS as well and we have a dense $G_{\delta}$ subset $\tilde{X}^{*} \subset \tilde{X}$ which makes $\tilde{X}$ AS.

Let $\left(\tilde{x}, \tilde{x}^{\prime}\right) \in S \cap\left(\tilde{X}^{*} \times \tilde{X}^{*}\right)$ and let $L=\bar{o}\left(\tilde{x}, \tilde{x}^{\prime}\right)$. By the AS property of $\tilde{X}$ there exists an almost automorphism $\psi$ with $\psi(\tilde{x})=\tilde{x}^{\prime}$. By Lemma $1.1 L[\tilde{x}]=\left\{\tilde{x}^{\prime}\right\}$ and by the definition of $S, L[\tilde{x}]=\tilde{\sigma}^{-1} \tilde{\sigma}\left(\tilde{x}^{\prime}\right)$. Thus $\tilde{\sigma}^{-1} \tilde{\sigma}(\tilde{x})=\left\{\tilde{x}^{\prime}\right\}$ and $\tilde{\sigma}$ is both open and almost $1-1$, hence an isomorphism. This means that $\sigma$ is almost $1-1$, proving our proposition.

4. The topological versions of Veech's theorem

The situation we consider here is described by the diagram

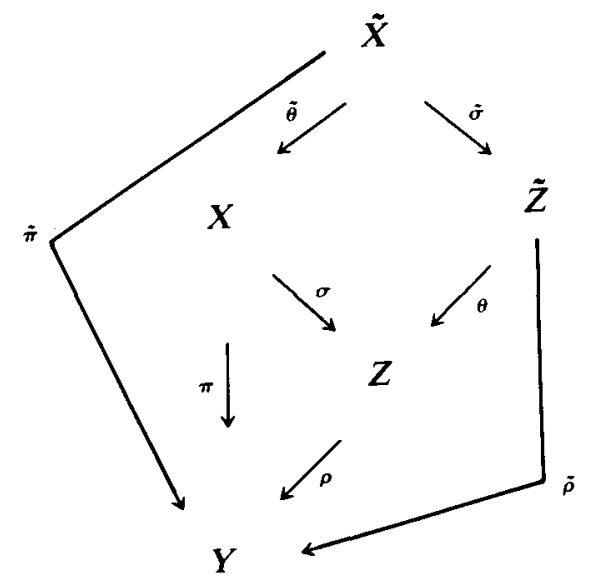


where $\pi$ is point distal open, $\rho$ is the largest almost periodic extension of $Y$ within $X$, and the top square is the shadow diagram for $\sigma$. Thus

$$
\begin{aligned}
& \tilde{Z}=\left\{p \circ \sigma^{-1}\left(z_{0}\right): p \in M\right\}, \quad \theta\left(p \circ \sigma^{-1}\left(z_{0}\right)\right)=p z_{0} \\
& \tilde{X}=\{(x, \tilde{z}): x \in \tilde{z} \in \tilde{Z}\}, \quad \tilde{\theta}(x, \tilde{z})=\tilde{z}, \quad \tilde{\sigma}(x, \tilde{z})=\tilde{z},
\end{aligned}
$$

$\theta, \tilde{\theta}$ are almost $1-1$ and $\tilde{\sigma}$ is open.

Let $Z_{0}=\left\{z \in Z:\left|\theta^{-1}(z)\right|=1\right\}, \tilde{Z}_{0}=\theta^{-1}\left(Z_{0}\right) \subset \tilde{Z}, X_{0}=\sigma^{-1}\left(Z_{0}\right), \tilde{X}_{0}=\tilde{\theta}^{-1}\left(X_{0}\right)$. Then $\tilde{X}_{0}=\left\{(x, \tilde{z}) \in \tilde{X}: \tilde{z} \in \tilde{Z}_{0}\right\}$ and the sets $Z_{0}, \tilde{Z}_{0}, X_{0}$ and $\tilde{X}_{0}$ are all dense $G_{\delta}$ subsets of the corresponding spaces.

Note that if $U$ is an open subset of $\tilde{X}$ then int $\tilde{\theta}(U) \supset \tilde{\theta}\left(\tilde{X}_{0} \cap U\right)$. To see this suppose $\tilde{x} \in \tilde{X}_{0} \cap U$ and $x_{i} \rightarrow x=\tilde{\theta}(\tilde{x})$. Then choosing $\tilde{x}_{i} \in \tilde{X}$ with $\tilde{\theta}\left(\tilde{x}_{i}\right)=x_{i}$ and choosing any subsequence $\tilde{x}_{i} \rightarrow \tilde{x}^{\prime}$ we have $\tilde{\theta}\left(\tilde{x}^{\prime}\right)=\tilde{\theta}(\tilde{x})$. Hence $\tilde{x}^{\prime}=\tilde{x}$ and therefore eventually $\tilde{x}_{i} \in U$. Thus $x_{i} \in \tilde{\theta}(U)$ and we conclude that $x \in \operatorname{int} \tilde{\theta}(U)$.

LEMMA 4.1. Let $X \stackrel{\pi}{\rightarrow} Y$ be open and point distal; then the set of almost periodic points in $R_{\pi}$ contains a dense $G_{\delta}$ subset $S_{1}$ of $R_{\pi}$. In particular $\pi$ is a Bc-extension.

Proof. By [E2] there exists a dense $G_{\delta}$ subset $X_{1}$ of $X$ consisting of $Y$-distal points. Since $\pi$ is open the projection maps $\pi_{j}: R_{n} \rightarrow X(j=1,2)$ are open and by Lemma 1.5 $S_{1}=\pi_{1}^{-1}\left(X_{1}\right) \cap \pi_{2}^{-1}\left(X_{1}\right)$ is a dense $G_{\delta}$ subset of $R_{\pi}$. If $\left(x, x^{\prime}\right) \in S_{1}$ and $v x=x$ for $v \in J$, then $v\left(x, x^{\prime}\right)=\left(x, v x^{\prime}\right) \in R_{\pi}$. Hence also $\left(x^{\prime}, v x^{\prime}\right) \in R_{\pi}$. But $x^{\prime}$ is $Y$-distal hence $x^{\prime}=v x^{\prime}$ so that $\left(x, x^{\prime}\right)$ is in $J R_{\pi}$.

LEMMA 4.2. The subset $S_{X}=\left(X_{0} \times X_{0}\right) \cap S_{1}$ is a dense $G_{\delta}$ subset of $R_{\pi}$.

Proof. As in the previous lemma $\left(X_{0} \times X_{0}\right) \cap R_{\pi}$ is dense $G_{\delta}$ in $R_{\pi}$ and hence so is $S_{X}=\left(X_{0} \times X_{0}\right) \cap R_{\pi} \cap S_{1}=\left(X_{0} \times X_{0}\right) \cap S_{1}$.

Since $\rho$ is almost periodic $R_{\rho}$ consists of almost periodic points and as above $S_{Z}=Z_{0} \times Z_{0} \cap R_{\rho}$ is a dense $G_{\delta}$ subset of $R_{\rho}$. Put $S_{\tilde{Z}}=\theta^{-1}\left(S_{Z}\right), S_{\tilde{X}}=\tilde{\theta}^{-1}\left(S_{X}\right)$ and $\tilde{S}_{\tilde{X}}=\tilde{\sigma}^{-1}\left(S_{\tilde{Z}}\right)$. Note that $S_{\tilde{Z}} \subset J R_{\tilde{\rho}}$ and $S_{\tilde{X}} \subset J R_{\tilde{\pi}} ;$ also $S_{\tilde{X}} \subset \tilde{S}_{\tilde{X}}$.

LeMMA 4.3. Let $V \subset R_{\tilde{\pi}}$ be open with $\tilde{\sigma}(V)=R_{\tilde{\rho}}$. Put $U=V \cap \overline{J R_{\tilde{\pi}}}$ then $U \cap S_{\tilde{X}}$ is dense in $V \cap \tilde{S}_{\tilde{X}}$.

Proof. Let $\left(\tilde{x}, \tilde{x}^{\prime}\right)=\left((x, \tilde{z}),\left(x^{\prime}, \tilde{z}^{\prime}\right)\right) \in V \cap \tilde{S}_{\tilde{X}}$, then there are open subsets $U, U^{\prime}$ of $\tilde{X}$ with $\left(\tilde{x}, \tilde{x}^{\prime}\right) \in\left(U \times U^{\prime}\right) \cap R_{\tilde{\pi}} \subset V$. Since $\left(x, x^{\prime}\right) \in X_{0} \times X_{0}$ we have $\left(x, x^{\prime}\right) \in$ [(int $\tilde{\theta}(U)) \times\left(\right.$ int $\left.\left.\tilde{\theta}\left(U^{\prime}\right)\right)\right] \cap R_{\pi}$. Therefore there are points $\left(x_{i}, x_{i}^{\prime}\right) \rightarrow\left(x, x^{\prime}\right)$ with $\left(x_{i}, x_{i}^{\prime}\right) \in S_{X} \cap\left[(\operatorname{int} \tilde{\theta}(U)) \times\left(\right.\right.$ int $\left.\left.\tilde{\theta}\left(U^{\prime}\right)\right)\right]$. Hence also $\left(\left(x_{i}, \tilde{z}_{i}\right), \quad\left(x_{i}^{\prime}, \tilde{z}_{i}^{\prime}\right)\right) \rightarrow((x, \tilde{z})$, $\left.\left(x^{\prime}, \tilde{z}^{\prime}\right)\right)=\left(\tilde{x}, \tilde{x}^{\prime}\right) \quad$ with $\quad\left(\left(x_{i}, \tilde{z}_{i}\right),\left(x_{i}, \tilde{z}_{i}^{\prime}\right)\right) \in S_{\tilde{X}} \cap V=S_{\tilde{X}} \cap J R_{\tilde{\pi}} \cap V \subset S_{\tilde{X}} \cap U$. Here $\left(\left(x_{i}, \tilde{z}_{i}\right),\left(x_{i}^{\prime}, \tilde{z}_{i}^{\prime}\right)\right)$ are the unique points in $R_{\tilde{\pi}}$ above $\left(x_{i}, x_{i}^{\prime}\right)$.

LEMMA 4.4. With $V$ and $U$ as in Lemma 4.3, if $\tilde{\sigma}(V)=R_{\dot{p}}$ then

$$
\overline{T U} \supset J R_{\tilde{\pi}} \text {. }
$$

Proof. Let $\left(\tilde{x}, \tilde{x}^{\prime}\right) \in J R_{\tilde{\pi}}$, say $v\left(\tilde{x}, \tilde{x}^{\prime}\right)=\left(\tilde{x}, \tilde{x}^{\prime}\right)$ with $v \in J$. Denote $\left(\tilde{z}, \tilde{z}^{\prime}\right)=$ $\tilde{\sigma}\left(\tilde{x}, \tilde{x}^{\prime}\right),\left(z, z^{\prime}\right)=\theta\left(\tilde{z}, \tilde{z}^{\prime}\right)$, then there exists a sequence $\left(z_{i}, z_{i}^{\prime}\right) \in S_{Z}$ such that $\left(z_{i}, z_{i}^{\prime}\right) \rightarrow$ $\left(z, z^{\prime}\right)$.

Let $\left(\tilde{z}_{i}, \tilde{z}_{i}^{\prime}\right) \in S_{\tilde{z}}$ be the unique point in $R_{\tilde{p}}$ with $\theta\left(\tilde{z}_{i}, \tilde{z}_{i}^{\prime}\right)=\left(z_{i}, z_{i}^{\prime}\right)$ and let $\left(\tilde{z}_{i}, \tilde{z}_{i}^{\prime}\right) \rightarrow$ $\left(\tilde{z}_{*}, \tilde{z}_{*}^{\prime}\right)$, then clearly $v\left(\tilde{z}_{*}, \tilde{z}_{*}^{\prime}\right)=\left(\tilde{z}, \tilde{z}^{\prime}\right)$. By assumption there are points $\left(\tilde{x}_{i}, \tilde{x}_{i}^{\prime}\right) \in V$ with $\tilde{\sigma}\left(\tilde{x}_{i}, \tilde{x}_{i}^{\prime}\right)=\left(\tilde{z}_{i}, \tilde{z}_{i}^{\prime}\right)$; let $\left(\tilde{x}_{i}, \tilde{x}_{i}^{\prime}\right) \rightarrow\left(\tilde{x}_{1}, \tilde{x}_{1}^{\prime}\right)$. 
Now $\left(\tilde{x}_{i}, \tilde{x}_{i}^{\prime}\right) \in \tilde{S}_{\tilde{X}} \cap V$ and by Lemma 4.3 we can find a sequence $\left(\bar{x}_{i}, \bar{x}_{i}^{\prime}\right) \in S_{\tilde{x}} \cap U$ such that $\left(\bar{x}_{i}, \bar{x}_{i}^{\prime}\right) \rightarrow\left(\tilde{x}_{1}, \tilde{x}_{1}^{\prime}\right)$. By Lemma 2.3

$$
\overline{T U} \supset\left(w_{i} \tilde{\sigma}^{-1} \tilde{\sigma}\left(\bar{x}_{i}\right)\right) \times\left(w_{i} \tilde{\sigma}^{-1} \tilde{\sigma}\left(\bar{x}_{i}\right)\right)
$$

where $w_{i} \in J$ satifies $w_{i}\left(\bar{x}_{i}, \bar{x}_{i}^{\prime}\right)=\left(\bar{x}_{i}, \bar{x}_{i}^{\prime}\right)$. As in the proof of Lemma 2.4 we also have $\overline{T U} \supset\left(w \tilde{\sigma}^{-1} \tilde{\sigma}\left(\tilde{x}_{1}\right)\right) \times\left(w^{\prime} \tilde{\sigma}^{-1} \tilde{\sigma}\left(\tilde{x}_{1}^{\prime}\right)\right)$ where $w, w^{\prime} \in J$ and $w \tilde{x}_{1}=\tilde{x}_{1}, w^{\prime} \tilde{x}_{1}^{\prime}=\tilde{x}_{1}^{\prime}$. It now follows that

$$
\begin{aligned}
\overline{T U} & \supset\left(v \tilde{\sigma}^{-1} \tilde{\sigma}\left(\tilde{x}_{1}\right)\right) \times\left(v \tilde{\sigma}^{-1} \tilde{\sigma}\left(\tilde{x}_{1}^{\prime}\right)\right) \\
& =\left(v \tilde{\sigma}^{-1}\left(z_{*}\right)\right) \times\left(v \tilde{\sigma}^{-1}\left(z_{*}^{\prime}\right)\right) \\
& =\left(v \tilde{\sigma}^{-1}(\tilde{z})\right) \times\left(v \tilde{\sigma}^{-1}\left(\tilde{z}^{\prime}\right)\right) \ni\left(\tilde{x}, \tilde{x}^{\prime}\right) .
\end{aligned}
$$

We can now prove the main theorems of this section.

TheOREM 4.5. Let $(X, T)$ be an AS flow and $(X, T) \stackrel{\pi}{\rightarrow}(Y, T)$ a non-trivial homomorphism. Then up to almost 1-1 equivalence, $\pi$ is a group extension.

Proof. Using a shadow diagram we can assume that $\pi$ is open and by Proposition 1.7 it is point distal. Construct the diagram @.

Let $\mathscr{V}=\left\{V \subset R_{\tilde{\pi}}: V\right.$ open, $\left.\tilde{\sigma}(V)=R_{\tilde{\rho}}\right\}$ and let $\left\{V_{i}\right\}_{i=1}^{\infty}$ be a basis for $\mathscr{V}$. For each $i$ let $U_{i}=V_{i} \cap \overline{J R_{\tilde{\pi}}}$. Then by Lemma 4.4 $T U_{i}$ is an open dense subset of $\overline{J R_{\tilde{\pi}}}$. Put

$$
S=\bigcap_{i=1}^{\infty} T U_{i}
$$

then $S$ is a dense $G_{\delta}$ subset of $\overline{J R_{\tilde{\pi}}}$. Now if $S_{X}$ is the subset of $J R_{\pi}$ defined in Lemma 4.2 and $X^{*}$ is a set which makes $X$ AS, then $S_{X}^{*}=\left(X^{*} \times X^{*}\right) \cap S_{X}$ is a dense $G_{\delta}$ subset of $J R_{\pi}$ and $\tilde{\theta}^{-1}: S_{X}^{*} \rightarrow J R_{\tilde{n}}$ is a homeomorphism. Denote $S_{\tilde{X}}^{*}=$ $\tilde{\theta}^{-1}\left(S_{X}^{*}\right)$, then $S_{\tilde{x}}^{*}$ is a dense $G_{\delta}$ subset of $\overline{J R_{\tilde{\pi}}}$. To see this let $\left(\tilde{x}, \tilde{x}^{\prime}\right)=\left((x, \tilde{z}),\left(x^{\prime}, \tilde{z}^{\prime}\right)\right) \epsilon$ $J R_{\tilde{\pi}}$, say $v\left(\tilde{x}, \tilde{x}^{\prime}\right)=\left(\tilde{x}, \tilde{x}^{\prime}\right)$. Then $\left(x, x^{\prime}\right) \in J R_{\pi}$ and there exists a sequence $\left(x_{i}, x_{i}^{\prime}\right) \in S_{X}^{*}$ converging to $\left(x, x^{\prime}\right)$. Let $\left(\tilde{x}_{i}, \tilde{x}_{i}^{\prime}\right)=\left(\left(x_{i}, \tilde{z}_{i}\right),\left(x_{i}^{\prime}, \tilde{z}_{i}^{\prime}\right)\right) \in S_{\tilde{x}}^{*}$ be the unique point of $R_{\tilde{\pi}}$ above $\left(x_{i}, x_{i}^{\prime}\right)$ and suppose $\left(\tilde{x}_{i}, \tilde{x}_{i}^{\prime}\right) \rightarrow\left(\tilde{x}_{1}, \tilde{x}_{1}^{\prime}\right)=\left(\left(x, \tilde{z}_{1}\right)\left(x^{\prime}, \tilde{z}_{1}^{\prime}\right)\right)$. Then clearly $v\left(\tilde{x}_{1}, \tilde{x}_{1}^{\prime}\right)=\left(\tilde{x}, \tilde{x}^{\prime}\right)$. Since $\left(\tilde{x}_{1}, \tilde{x}_{1}^{\prime}\right) \in S_{\tilde{x}}^{*}$ and since the latter set is $T$-invariant and closed it also contains $\left(\tilde{x}, \tilde{x}^{\prime}\right)$. Thus $S_{\tilde{x}}^{*}$ is dense $G_{\delta}$ in $\overline{J R_{\tilde{\pi}}}$ and therefore $S_{\tilde{x}}^{*} \cap S$ is non-empty. Let $\left(\tilde{x}, \tilde{x}^{\prime}\right) \in S_{\tilde{X}}^{*} \cap S$ then $\tilde{x}^{\prime}=\psi(\tilde{x})$ for an almost automorphism $\psi$ and in particular $L=\bar{o}\left(\tilde{x}, \tilde{x}^{\prime}\right)$ is minimal. If there exists a $\left(\tilde{z}, \tilde{z}^{\prime}\right) \in R_{\tilde{\rho}}$ with

$$
\tilde{\sigma}^{-1}\left(\tilde{z}, \tilde{z}^{\prime}\right) \subset L
$$

then we have (by minimality of $L$ and openness of $\tilde{\sigma}$ )

$$
R_{\tilde{\sigma}} \circ L \circ R_{\tilde{\sigma}} \subset L
$$

and $L[\tilde{x}]=\left\{\tilde{x}^{\prime}\right\}=R_{\tilde{\sigma}}\left[\tilde{x}^{\prime}\right]$ so that $\tilde{\sigma}$ is both open and almost 1-1. Hence $\tilde{\sigma}$ is an isomorphism and our theorem follows from Proposition 1.9. If no such $\left(\tilde{z}, \tilde{z}^{\prime}\right)$ exists then $V=R_{\tilde{\pi}} \backslash L$ is open and satisfies $\tilde{\sigma}(V)=R_{\tilde{\rho}}$, i.e. $V \in \mathscr{V}$. Choose an $i$ with $V_{i} \subset V$, then $\exists t \in T$ with $t\left(\tilde{x}, \tilde{x}^{\prime}\right) \in V_{i} \subset V$, a contradiction. This completes the proof.

We actually proved the following version of Proposition 2.5.

Proposition 4.6. Consider the diagram (@) where we now assume that $\pi$ is open point distal, $\rho$ the maximal almost periodic extension of $Y$ within $X$ and $\tilde{\sigma}$ the open shadow map of $\sigma$. Then there exists a dense $G_{\delta}$ subset $S$ of $\overline{J R_{\tilde{\pi}}}$ such that for $\left(\tilde{x}, \tilde{x}^{\prime}\right) \in S$ $\bar{o}\left(\tilde{x}, \tilde{x}^{\prime}\right)$ is $R_{\tilde{\sigma}}$ saturated. 
THEOREM 4.7. Let $(X, T)$ be a minimal metric flow and let $X \stackrel{\pi}{\rightarrow} Y$ be a regular point distal, open homomorphism. Then $\pi=\rho \circ \sigma$ where $\rho$ is a group extension and $\sigma$ almost 1-1. In particular a metric regular point distal flow is equicontinuous.

Proof. Construct the diagram (@) as in the proof of Theorem 4.5. Let $\mathscr{V},\left\{V_{i}\right\}_{i=1}^{\infty}$, $\left\{U_{i}\right\}_{i=1}^{\infty}$ and $S$ be defined in the same way. Let $S_{X}$ be as in Lemma 4.2 , put $S_{\tilde{X}}=\tilde{\theta}^{-1}\left(S_{X}\right)$, choose $\left(\tilde{x}, \tilde{x}^{\prime}\right) \in S_{\tilde{X}} \cap S$ and let $L=\tilde{o}\left(\tilde{x}, \tilde{x}^{\prime}\right)$. Since $\left(\tilde{x}, \tilde{x}^{\prime}\right) \in S_{\tilde{X}}, L$ is minimal and $\theta\left(\tilde{x}, \tilde{x}^{\prime}\right)=\left(x, x^{\prime}\right)$ is an almost periodic point in $R_{\pi}$. By regularity of $\pi$, $x^{\prime}=\psi(x)$ for some $\psi \in$ Aut $(X, T)$. Lifting $\psi$ to $\tilde{X}_{0}$ we have $\tilde{x}^{\prime}=\psi(\tilde{x})$ and we finish the proof as in Theorem 4.5. However, since our $\pi$ was assumed to be open we actually get that $\pi=\rho \circ \sigma$ where $\rho$ is a group extension and $\rho$ is almost $1-1$. The last part of the theorem follows from Proposition 1.10.

Problem. Does there exist an AS weakly mixing flow $X$ which is an inverted tower of group extensions. I.e., $X \stackrel{\pi_{n}}{\rightarrow} X_{n}$ is a group extension, $R_{\pi_{n+1}} \supset R_{\pi_{n}}$ and $\bigcup_{n=1}^{\infty} R_{n}$ is dense in $X \times X$ ? Can any weakly mixing minimal flow have such a structure? (See [G-W] for a weaker result.)

\section{Preliminary lemmas}

Our purpose in the rest of this paper is to deal with minimal joinings of AS flows.

LEMMA 5.1. Let $X$ and $Y$ be minimal flows and $L \subset Y \times X$ a minimal subset. There exist an almost 1-1 extension $\tilde{Y} \rightarrow Y$ and a minimal subset $\tilde{L} \subset \tilde{Y} \times X$ projecting onto $L$, such that the projection of $\tilde{L}$ onto $\tilde{Y}$ is an open homomorphism.

Proof. Let $\pi: L \rightarrow Y$ be the projection and let $L[y]=\{x \in X:(y, x) \in L\}$. Since $\pi^{-1}: Y \rightarrow 2^{L}$ is an upper semicontinuous map it has a dense $G_{\delta}$ subset $Y_{0} \subset Y$ of continuity points. Choose $y_{0} \in Y_{0}$ and let $\tilde{y}_{0}=\pi^{-1}\left(y_{0}\right)=\left\{y_{0}\right\} \times L\left[y_{0}\right] \in 2^{L}$. Define $\tilde{Y}=\bar{o}\left(\tilde{y}_{0}\right)=\left\{p \circ \pi^{-1}\left(y_{0}\right): p \in M\right\}=\left\{\left\{p y_{0}\right\} \times\left(p \circ L\left[y_{0}\right]\right): p \in M\right\}$. We denote by $\varphi$ the canonical map of $\tilde{Y}$ onto $Y$ (projection on the first coordinate). Finally, let $\tilde{L}=$ $\{(\tilde{y}, x):(\varphi(\tilde{y}), x) \in \tilde{y} \in \tilde{Y}\}$.

If $\tilde{y}_{i} \rightarrow \tilde{y}$ is a convergent sequence in $\tilde{Y}$ and $(\tilde{y}, x) \in \tilde{L}$ then $(\varphi(\tilde{y}), x) \in \tilde{y}$ and by the definition of the topology in $2^{\mathrm{L}}$ it follows that a sequence $\left(y_{i}, x_{i}\right) \in \tilde{y}_{i}$ exists with $\left(y_{i}, x_{i}\right) \rightarrow(\varphi(\tilde{y}), x)$. This shows that the projection $\tilde{\pi}: \tilde{L} \rightarrow \tilde{Y}$ is open.

For a point $y \in Y_{0}$ and $p \in M$ with $p y_{0}=y$ we have $p\left(\left\{y_{0}\right\} \times L\left[y_{0}\right]\right)=\{y\} \times L[y]=\tilde{y}$. It follows that $\varphi^{-1}(y)=\{\tilde{y}\}$ and $\varphi$ is almost $1-1$.

By definition $\tilde{Y}$ is minimal. To see that $\tilde{L}$ is minimal choose a point $\left(y_{0}, x_{0}\right) \in \dot{y}_{0}=$ $\left\{y_{0}\right\} \times L\left[y_{0}\right]$ so that $\left(\tilde{y}_{0}, x_{0}\right)$ is an almost periodic point of $\tilde{L}$. If $\left(y_{0}, x\right) \in \tilde{y}_{0}$ then $\left(y_{0}, x\right) \in L$ and there exists a sequence $t_{i} \in T$ such that $t_{i}\left(y_{0}, x_{0}\right) \rightarrow\left(y_{0}, x\right)$. By continuity of $\pi^{-1}$ at $y_{0}$ also $t_{i} \tilde{y}_{0} \rightarrow \tilde{y}_{0}$ so that $\left(\tilde{y}_{0}, x\right) \in \bar{o}\left(\tilde{y}_{0}, x_{0}\right)$. If now $(\tilde{y}, x)$ is an arbitrary point of $\tilde{L},(\varphi(\tilde{y}), x) \in \tilde{y}$ implies that a sequence $t_{i} \in T$ exists that $\lim t_{i} \tilde{y}_{0}=\tilde{y}$ and $x=\lim t_{i} x_{i}$ where $\left(y_{0}, x_{i}\right) \in \tilde{y}_{0}$.

Thus $(\tilde{y}, x)=\lim t_{i}\left(\tilde{y}_{0}, x_{i}\right) \in \bar{o}\left(\tilde{y}_{0}, x_{0}\right)$ and $\tilde{L} \subset \bar{o}\left(\tilde{y}_{0}, x_{0}\right)$ is minimal.

When $L \subset Y \times X$ is minimal and the projection $\pi: L \rightarrow Y$ is open, the map $y \mapsto L[y]$ from $Y$ into $2^{X}$, is a homomorphism whose image $\{L[y]: y \in Y\}$ is a quasifactor of $X$. If we change notation and call this quasifactor $Y$ then we are at the situation 
assumed in the next lemma. This lemma is a version of a topological 'Fubini theorem' of the type proved by Kuratowski and Ulam. We follow Veech [V3, Proposition 3.1].

LeмmA 5.2. Let $(X, T)$ be a minimal flow, $Y \subset 2^{X}$ a minimal quasi-factor such that

$$
L=\{(y, x): x \in y \in Y\} \subset Y \times X
$$

is minimal. Let $X^{*} \subset X$ be a dense $G_{\delta}$ subset of $X$, then

$$
Y^{*}=\left\{y \in Y: y \cap X_{0} \text { is dense } G_{\delta} \text { in } y\right\}
$$

is a residual subset of $Y$.

Proof. Let $X^{*}=\bigcap_{l=1}^{\infty}, V_{l}$ where $\left\{V_{l}\right\}_{l=1}^{\infty}$ are open dense subsets of $X$. Let $\left\{U_{m}\right\}_{m=1}^{\infty}$ be a basis for the topology on $X$. A point $y \in Y$ is not in $Y^{*}$ iff there are $m$ and $l$ such that $y \in C_{m, l}$ where

$$
C_{m, l}=\left\{z \in Y: \phi \neq z \cap U_{m} \subset V_{l}^{c}\right\} .
$$

Denote $E_{m, l}=\operatorname{int}\left(\bar{C}_{m, l}\right)$ and suppose $E_{m, l} \neq \varnothing$. Let $\pi_{i}(i=1,2)$ be the projections of $L$ on $Y$ and $X$ respectively. Then $V=\pi_{1}^{-1}\left(E_{m, l}\right) \cap \pi_{2}^{-1}\left(U_{m}\right)$ is a non-empty open subset of $L$ because $C_{m, l} \cap E_{m, l}$ is dense in $E_{m, l}$ and $z \in C_{m, l}$ implies $z \cap U_{m} \neq \varnothing$.

Since $L$ is minimal int $\pi_{2}(V)=U$ is an open non-empty subset of $X$. We claim that $U \subset V_{l}^{c}$. This contradiction (we assumed $V_{l}$ is open dense) will show that $E_{m, l} \neq \varnothing$ and it will follow that $\left(Y^{*}\right)^{c}$ is of first category.

Let $x \in U$ say $x=\pi_{2}(y, x),(y, x) \in V$. Since $C_{m, l} \cap E_{m, l}$ is dense in $E_{m, l}, \pi_{1}^{-1}\left(C_{m, l} \cap\right.$ $\left.E_{m, l}\right)$ is dense in $\pi_{1}^{-1}\left(E_{m, l}\right)$ (use again the minimality of $L$ ), and $\pi_{1}^{-1}\left(C_{m, l} \cap E_{m, l}\right) \cap$ $\pi_{2}^{-1}\left(U_{m}\right)$ is dense in $V$. Thus we can find a sequence $\left(y_{i}, x_{i}\right) \rightarrow(y, x)$ such that $y_{i} \in C_{m, l} \cap E_{m, l}, x_{i} \in U_{m}$ and $\left(y_{i}, x_{i}\right) \in L$. By definition of $C_{m, l}$ it follows that $x_{i} \in V_{i}^{c}$ and since the latter set is closed, also $x \in V_{l}^{c}$. We have shown that $U \subset V_{l}^{c}$ and the proof is completed.

Remark. The use we made of the group action here, was only to insure that the maps $\pi_{i}(i=1,2)$ are semi-open. Taking $Y \subset 2^{X}$ to be a closed subset and assuming the semi-openness of $\pi_{i}(i=1,2)$ our lemma becomes purely topological (no dynamics is involved).

LEMMA 5.3. Let $\tilde{Y}$ be a quasifactor of $X$ then the sets

$$
\begin{aligned}
& \tilde{Y}_{\max }=\{\tilde{y} \in \tilde{Y}: \tilde{y} \subset \tilde{z} \in \tilde{Y} \Rightarrow \tilde{y}=\tilde{z}\} \\
& \tilde{Y}_{\min }=\{\tilde{y} \in \tilde{Y}: \tilde{y} \supset \tilde{z} \in \tilde{Y} \Rightarrow \tilde{y}=\tilde{z}\}
\end{aligned}
$$

and therefore also $\tilde{Y}_{m}=\tilde{Y}_{\max } \cap \tilde{Y}_{\min }$ are dense $G_{\delta}$ subsets of $\tilde{Y}$.

Proof. By Zorn's lemma $\tilde{Y}_{\max }$ is non-empty; by minimality of $\tilde{Y}$ and invariance it is dense. Finally

$$
F_{\varepsilon}=\{\tilde{y} \in \tilde{Y}: \exists \tilde{z} \in \tilde{Y}, \tilde{z} \supset \tilde{y}, d(\tilde{z}, \tilde{y}) \geq \varepsilon\}
$$

is clearly closed so that

$$
\tilde{Y}_{\max }=\bigcap_{n=1}^{\infty}\left(F_{1 / n}\right)^{c}
$$

is dense $G_{\delta}$. The proof for $\tilde{Y}_{\min }$ is analogous. 


\section{The joinings of an AS flow}

We say that a property of minimal flows essentially holds if it holds up to almost 1-1 extensions. For example $X$ is essentially an extension of $Y$ if an almost 1-1 extension of $X$ is an extension of $Y$. The flows $X$ and $Y$ have essentially no common factor if no almost 1-1 extension of $X$ and no such extension of $Y$ have a non-trivial common fctor. It is easy to see that $X$ and $Y$ are essentially disjoint iff they are disjoint.

THEOREM 6.1. Suppose $T$ is a commutative group. Let $(X, T)$ be an AS flow, $(Y, T)$ a minimal flow and $L \subset Y \times X$ a minimal subset such that $L \neq Y \times X$. Then up to almost 1-1 equivalence $L$ is the relative product of $X$ and $Y$ over a common factor which is essentially a group factor of $X$.

Corollaries 6.2. For commutative $T$

(1) If $(X, T)$ is AS and purely weakly mixing, then $X$ is disjoint from every minimal flow which is not an essential extension of it.

(2) If ( $X, T)$ is AS and ( $Y, T)$ minimal, then they are disjoint iff they have essentially no common factors.

Proof of Theorem 6.1. Let $L \subset Y \times X$ be minimal and we assume $L \neq Y \times X$. Let $\pi_{1}: L \rightarrow Y$ and $\pi_{2}: L \rightarrow X$ be the projections. By Lemma 5.1 we can assume that $\pi_{1}$ is open. This implies that the map $\gamma: Y \rightarrow 2^{X}$ defined by $\gamma(y)=L[y]$, is a continuous homomorphism. We denote by $\tilde{Y}$ the quasifactor $\{L[y]: y \in Y\}$ which is the image of $\gamma$ in $2^{x}$.

Define $W=\left\{\left(x_{1}, x_{2}\right) \in X^{2}: \exists y \in Y,\left(y, x_{i}\right) \in L, i=1,2\right\}$. We let $L^{*}=L \cap\left(Y \times X^{*}\right)$, $W^{*}=W \cap\left(X^{*} \times X^{*}\right)$, and $\Psi=\left\{\psi: \psi\right.$ is an almost automorphism of $(X, T), D_{\psi}$, $\psi\left(D_{\psi}\right) \subset X^{*}$ and for some $\left.x \in D_{\psi},(x, \psi(x)) \in W^{*}\right\}$.

LEMMA 6.3. For commutative $T, W \neq X \times X$.

Proof. If $W=X \times X$ then for all $t \in T$ and $x \in X,(x, t x) \in W$ so that given $(y, x) \in L$ also $(I \times t)(y, x)=(y, t x) \in L$. Since $L$ is minimal this implies $(I \times t) L=L \quad \forall t \in T$ and since $(X, T)$ is minimal we get $L=Y \times X$ contradicting our assumption.

LEMMA 6.4. If $W \neq X \times X$ then for every $y \in Y$ for which $L^{*}[y] \neq \emptyset$ and every $x \in L^{*}[y]$, $L^{*}[y]=\left\{\psi(x): \psi \in \Psi\right.$ and $\left.x \in D_{\psi}\right\}$. Thus for $y_{1}, y_{2} \in Y$ with $L^{*}\left[y_{i}\right] \neq \emptyset, i=1,2$, either $L^{*}\left[y_{1}\right]=L^{*}\left[y_{2}\right]$ or $L^{*}\left[y_{1}\right] \cap L^{*}\left[y_{2}\right]=\emptyset$.

Proof. Let $x, x^{\prime} \in L^{*}[y]$ then $\left(x, x^{\prime}\right) \in W^{*}$ and since $\bar{o}\left(x, x^{\prime}\right) \subset W \neq X \times X$ we have by AS $x^{\prime}=\psi(x)$ for an almost automorphism $\psi \in \Psi$. Conversely, suppose $\psi \in \Psi$ and $x \in L^{*}[y] \cap D_{\psi}$.

By definition of $\Psi$ there exists $\bar{x} \in D_{\psi}$ with $(\bar{x}, \psi(\bar{x})) \in W^{*}$ so that for some $\bar{y}=Y$, $(\bar{y}, \bar{x}),(\bar{y}, \psi(\bar{x})) \in L^{*}$. By minimality of $L$ there exists $t_{i} \in T$ with $(y, x)=\lim t_{i}(\bar{y}, \bar{x})$. Now

$$
\begin{aligned}
(y, \psi(x)) & =(I \times \psi)(y, x)=(I \times \psi) \lim t_{i}(\bar{y}, \bar{x}) \\
& =\lim t_{i}((I \times \psi)(\bar{y}, \bar{x}))=\lim t_{i}(\bar{y}, \psi(\bar{x})) \in L .
\end{aligned}
$$

Since by assumption $\psi\left(D_{\psi}\right) \subset X^{*}$ we also have $(y, \psi(x)) \in L^{*}$, i.e. $\psi(x) \in L^{*}[y]$. 
LEMMA 6.5. Assume $T$ is commutative, then the quasifactor $\tilde{Y}=\{L[y]: y \in Y\}$ of $X$, is a factor of an almost 1-1 extension of $X$.

Proof. By Lemma 5.2 the set $\tilde{Y}^{*}=\left\{\tilde{y} \in \tilde{Y}: \tilde{y} \cap X^{*}\right.$ is dense $G_{\delta}$ in $\left.\tilde{y}\right\}$ is dense $G_{\delta}$ in $\tilde{Y}$. By Lemma $5.3 \tilde{Y}_{m}=\tilde{Y}_{\max } \cap \tilde{Y}_{\min }$ is also dense $G_{\delta}$ in $\tilde{Y}$. Let $y_{0} \in Y$ be such that $L\left[y_{0}\right]=\tilde{y}_{0}$ is in $\tilde{Y}^{*} \cap \tilde{Y}_{m}$. Let $x_{0} \in L^{*}\left[y_{0}\right]$, then $\left(L\left[y_{0}\right], x_{0}\right)=\left(\tilde{y}_{0}, x_{0}\right)$ is an almost periodic point of $\tilde{Y} \times X$. In fact $\left(y_{0}, x_{0}\right) \in L$ implies that $\left(y_{0}, x_{0}\right)$ is an almost periodic point and if $v \in J$ is such that $v\left(y_{0}, x_{0}\right)=\left(y_{0}, x_{0}\right)$ then $v\left(\tilde{y}_{0}, x_{0}\right)=\left(v \circ L\left[y_{0}\right], x_{0}\right)=$ $\left(L\left[y_{0}\right], x_{0}\right)=\left(\tilde{y}_{0}, x_{0}\right)$, so that $\left(\tilde{y}_{0}, x_{0}\right)$ is also almost periodic. Define $\tilde{X}=\bar{o}\left(\tilde{y}_{0}, x_{0}\right)$ and $\lambda: L \rightarrow \tilde{X}$ by $\lambda(y, x)=\lambda\left(p y_{0}, p x_{0}\right)=\left(p \circ L\left[y_{0}\right], p x_{0}\right)=(L[y], x)$, where $(y, x)=$ $p\left(y_{0}, x_{0}\right)(p \in M)$. Let $\varphi: \tilde{X} \rightarrow X$ be the projection on the second coordinate. Suppose $\varphi\left(L[y], x_{0}\right)=x_{0},\left(L[y], x_{0}\right) \in \tilde{X}$. Then $x_{0} \in L^{*}[y]$ and by Lemmas 6.3 and $6.4 L^{*}[y]=$ $L^{*}\left[y_{0}\right]$. Since $\tilde{y}_{0} \in \tilde{Y}^{*}$ we have $L\left[y_{0}\right]=L^{*}\left[y_{0}\right]$, thus $L\left[y_{0}\right] \subset L[y]$. However $\tilde{y}_{0} \in \tilde{Y}_{\max }$ and therefore $L\left[y_{0}\right]=L[y]$. This shows that $\varphi^{-1}\left(x_{0}\right)=\left\{\left(\tilde{y}_{0}, x_{0}\right)\right\}$ and $\varphi$ is almost 1-1. The projection $\tilde{\pi}$ on the first coordinate is the required map from $\tilde{X}$ to $\tilde{Y}$.

Under the assumptions of Theorem 1.1 and the additional assumption that $\pi_{1}: L \rightarrow Y$ is open (we may assume this by Lemma 5.1) we obtained the following diagram

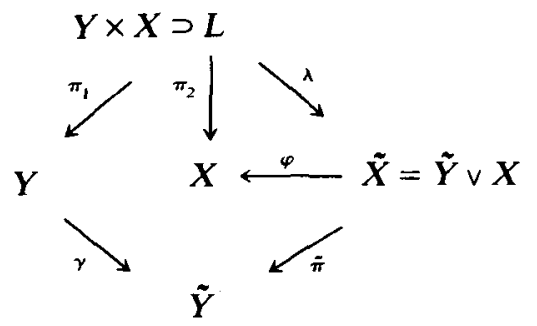

Here $\varphi$ is almost $1-1, \gamma$ is the map $y \mapsto L[y], \tilde{Y}$ is the quasifactor $\{L[y]: y \in Y\}$ of $X, \tilde{\pi}$ is the projection of $\tilde{X}$ onto $\tilde{Y}$ and $\lambda$ is the map defined by $\lambda(y, x)=(L[y], x)$ in the proof of Lemma 6.5 .

It is now clear that $L$ is the relative product of $Y$ and $\tilde{X}$ over $\tilde{Y}$. By Theorem $4.5 \tilde{\pi}$ is, up to almost $1-1$ extensions, a group extension. This completes the proof of Theorem 6.1.

Corollaries 6.2.1 and 6.2.2 now follow from the definitions of the relevant notions. We remark that the only place where the commutativity of $T$ was used in the proof of Theorem 6.1 is at the proof of Lemma 6.3, where we use the fact that each $t \in T$ defines an automorphism of $(X, T)$. Thus for a general group $T$ our theorem still holds if we know that $W \neq X \times X$.

Problem. Is the assumption that $T$ is abelian in Theorem 6.1 necessary?

Acknowledgment. I wish to thank N. Markley and J. Auslander for their helpful comments on a first draft of this paper. 


\section{REFERENCES}

[A] J. Auslander. Endomorphisms of minimal sets. Duke Math. J. 30 (1963), 605-614.

[A1] J. Auslander. Minimal Flows and Their Extensions. Math. Studies 153, North-Holland: Amsterdam, 1988.

[B] I. U. Bronšteìn. Extensions of Minimal Transformation Groups. Sijthoff \& Noordhoff: 1979.

[E1] R. Ellis. Lectures on Topological Dynamics. Benjamin: New York, 1969.

[E2] R. Ellis. The Veech structure theorem. Trans. Amer. Math. Soc. 186 (1973), 203-218.

[E-G] R. Ellis \& S. Glasner. Pure weak mixing. Trans. Amer. Math. Soc. 243 (1978), 135-146.

[G1] S. Glasner. Proximal flows. Lecture Notes in Math. 517. Springer Verlag: New York, 1976.

[G-W] S. Glasner \& B. Weiss. A weakly mixing upside down tower of isometric extensions. Ergod. Th. \& Dynam. Sys. 1 (1981), 151-157.

[Go] W. H. Gottschalk. Transitivity and equicontinuity. Bull. Amer. Math. Soc. 54 (1948), $982-984$.

[J] A. del Junco. On minimal self-joinings in topological dynamics. Ergod. Th. \& Dynam. Sys. 7 (1987), 211-227.

[J-R] A. del Junco \& D. Rudolph. On ergodic actions whose self-joinings are graphs. Ergod. Th. \& Dynam. Sys. 7 (1987), 531-557.

[V1] W. A. Veech. Topological Dynamics, Bull. Amer. Math. Soc. 83 (1977), 775-830.

[V2] W. A. Veech. A criterion for a process to be prime. Monat. Math. 94 (1982), 335-341.

[V3] W. A. Veech. Point-distal flows. Amer. J. Math. 92 (1970), 205-242. 